\title{
Perspective
}

PERSPECTIVE Actualité en histoire de l'art

$1 \mid 2020$

Japon

\section{Un cosmoscape sous le Bouddha : le piédestal de l'icône principale de Yakushi-ji, soutien de l'empire des souverains}

Cosmoscape Beneath the Buddha: Upholding the Realm of the Sovereigns on the Pedestal of Yakushi-ji's Main Icon

Ein Kosmoslandschaft unter dem Buddha: Der Sockel der Hauptikone von

Yakushi-ji als Stütze des Herrscherreichs

Uno cosmoscape ai piedi di Buddha: il piedistallo dell'icona principale di

Yakushi-ji, sostegno dell'impero dei sovrani

Cosmoscape bajo el Buda: el pedestal del principal icono de Yakushi-ji, sostén

del imperio de los soberanos

\section{Cynthea J. Bogel}

Traducteur : Étienne Gomez

\section{(2) OpenEdition}

\section{Journals}

\section{Édition électronique}

URL : http://journals.openedition.org/perspective/18208

DOI : 10.4000/perspective. 18208

ISSN : 2269-7721

\section{Éditeur}

Institut national d'histoire de l'art

\section{Édition imprimée}

Date de publication : 5 juin 2020

Pagination : 141-166

ISBN : 978-2-917902-89-9

ISSN : $1777-7852$

Référence électronique

Cynthea J. Bogel, « Un cosmoscape sous le Bouddha : le piédestal de l'icône principale de Yakushi-ji soutien de l'empire des souverains », Perspective [En ligne], 1 | 2020, mis en ligne le 30 décembre 2020, consulté le 25 janvier 2021. URL : http://journals.openedition.org/perspective/18208 ; DOI : https://doi.org/10.4000/perspective.18208 


\section{Un cosmoscape sous le Bouddha : le piédestal de l'icône principale de Yakushi-ji, soutien de l'empire des souverains}

Cynthea J. Bogel

\section{Représenter la souveraineté}

Le honzon 本尊 ( «icône principale ») du temple Yakushi-ji 薬師寺 de Nara 奈良, capitale japonaise de 710 à $785^{1}$, est composé d'un Bouddha de la Médecine assis en bronze coulé (Bouddha de Yakushi-ji ou Yakushi Nyorai 薬師如来; sk. Bhaiṣajyaguru Tathāgata) et de ses deux bodhisattva Nikkō et Gakkō̄ (fig. 1). Il est aujourd'hui exposé dans le kondō 金堂 ( ( pavillon principal »), un grand bâtiment achevé en 1976 à peu près au même emplacement que celui du VIII ${ }^{\mathrm{e}}$ siècle. Les spécialistes le font remonter à la période d'Asuka 飛鳥 (538-710) ou, selon la précision en usage principalement chez les historiens de l'art, à la période de Hakuhō 白鳳 (645710). Le débat sur sa date en recouvre un second, sur son origine, dans lequel s'inscrit l'article qui suit. D'une teinte noirâtre, il était autrefois doré à la feuille d'or, comme le montrent encore aujourd'hui des traces sur son piédestal ${ }^{3}$. Par sa taille colossale $(254,7 \mathrm{~cm})$, ce Bouddha peut être qualifié de jōrok $u^{4}$, une hauteur en usage pour les icônes de temples importants, soutenus par des fonds impériaux.

Si cette triade témoigne d'une grande valeur artistique et d'une immense prouesse technique, c'est le piédestal du Bouddha (fig. 2) qui suscite une intense spéculation dans les études japonaises, avec sa composition étonnante - et virtuose - de figures et de symboles en relief sur toutes ses faces. Il s'agit ici de situer ce piédestal dans la perspective de l'histoire du
1. Triade du Bouddha de Yakushi-ji ou Yakushi Nyorai [Bouddha de la Médecine], Nara, kondō du temple Yakushi-ji. Issu de Mizuno Keizaburō, Sekiguchi Kin'ya, Ōnishi Shūya (dir.), Hōryū-ji kara Yakushiji e: Asuka, Nara no kenchiku, chōkoku, Nihon bijutsu zenshū, Tōkyō, Kōdansha, 1990, vol. 2, pl. 140.

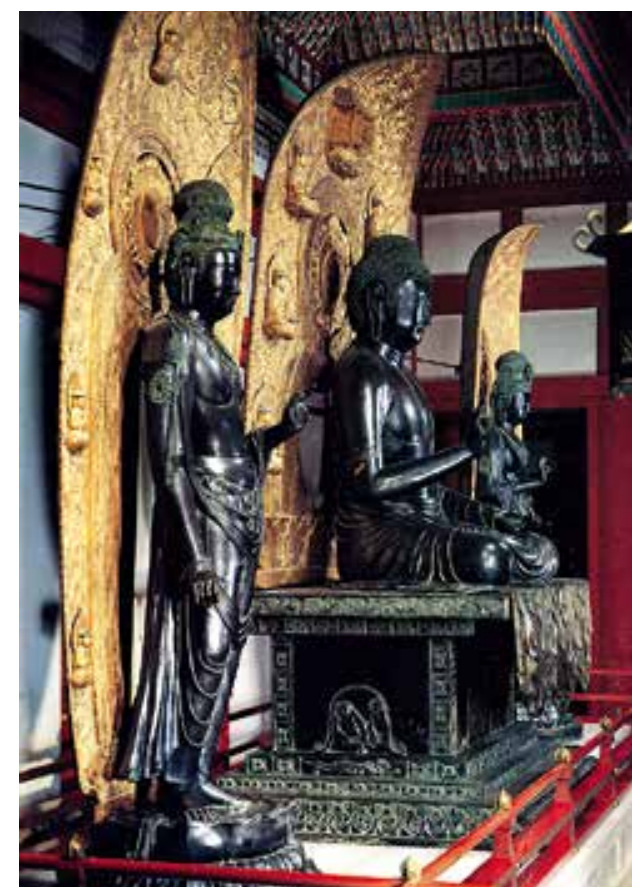




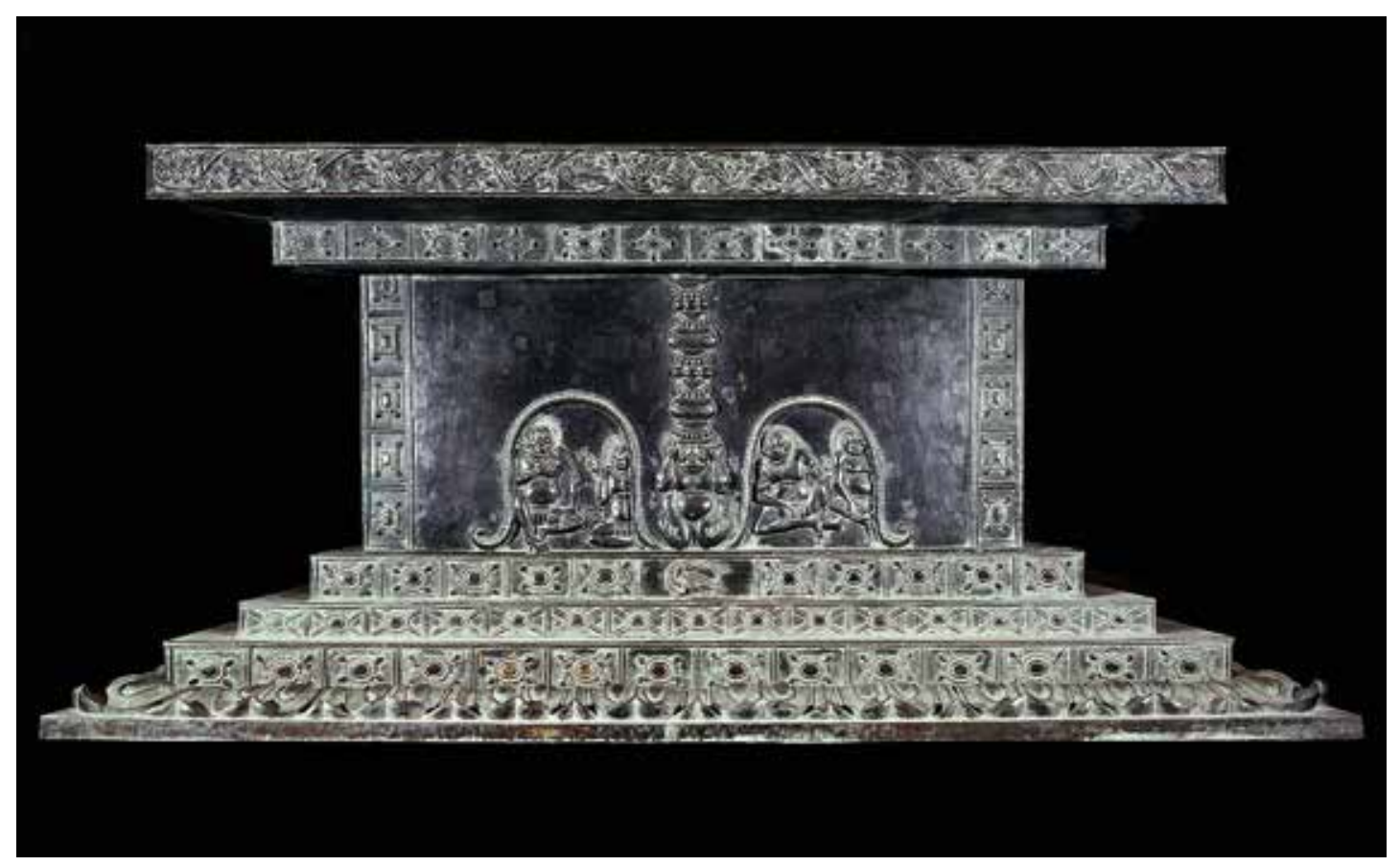

2. Piédestal du Yakushi Nyorai [Bouddha de la Médecine], face nord (arrière), dernier quart du vII siècle - première moitié du VIII ${ }^{\mathrm{e}}$ siècle, bronze avec des vestiges de feuille d'or, Nara, kondō du temple Yakushi-ji. temple et de ses fondateurs de façon à établir l'origine de l'icône ainsi que son rôle dans l'élaboration à la fois de l'histoire de cette période et de cosmologies en lien avec la souveraineté. J'appellerai cosmoscape le mode d'expression particulier qui s'y déploie.

La triade du Bouddha de Yakushi-ji a été déclarée Trésor national en 1951, et le temple a été inscrit au patrimoine mondial de l'UNESCO ( " Monuments

historiques de l'ancienne Nara ") en $1998^{5}$. Plus d'une centaine d'articles et d'ouvrages académiques en japonais, dont des catalogues de musée, ont été consacrés à l'archéologie et à la culture visuelle de Yakushi-ji depuis les années 1940, mais, à ma connaissance, aucune étude approfondie n'a été publiée à l'étranger ${ }^{6}$. Tout en se rattachant à cette riche historiographie, je souhaite développer une hypothèse originale sur l'iconographie du piédestal, en lien avec les mécènes impériaux du temple ainsi qu'avec l'histoire et la littérature contemporaines de sa création. Le piédestal apparaît comme un témoignage et comme un instrument de l'élaboration d'un modèle de gouvernement et d'une idéologie impériale de style chinois, réunissant des concepts, des figures et des symboles représentatifs d'un imaginaire centré autour de la cour, un peu de la même manière que des œuvres littéraires et historiographiques majeures comme le Kojiki 古事記 (compilé en 712), le Nihon shoki 日本 書紀 (720), les Fudoki 風土記 (731), le Kaifūsō 懐風藻 (751), ou encore le Man'yōshū 万葉集 (VIII ${ }^{\text {e }}$ siècle). Le processus de composition ou de compilation de ces œuvres littéraires date des mêmes décennies que la fondation de Yakushi-ji et la création de son honzon.

Tout comme ces œuvres littéraires, Yakushi-ji forme un ensemble composite. Fondé en 680 suite au vou de l'empereur, Tenmu Tennō 天武天皇 (631 ?-686 ; r. 673-686), pour la guérison de la princesse consort, le temple a été construit dans la partie occidentale de la ville aujourd'hui appelée Fujiwarakyō 藤原京 (694-710), la première à avoir été construite sur un plan en grille, dans le style chinois ${ }^{7}$. L'épouse de Tenmu Tennō fut guérie, 
et ce fut elle qui, après la mort de l'empereur, conduisit à son terme la construction du " temple du Bouddha de la Médecine " qui avait veillé à sa guérison. Selon Wadō 和銅 1 (708).2.15, Genmei Tennō 元明天皇 (661-721；r. 707-715) annonça ensuite par rescrit impérial le transfert du palais de Fujiwarakyō à Nara, qui, entrepris en 710, fut suivi du transfert de plusieurs temples. Les témoignages permettent de dater celui de Yakushi-ji de 718. Aujourd'hui le temple de Fujiwarakyō est appelé Moto Yakushi-ji 本薬師寺 ( "Yakushi-ji premier ») pour le distinguer du temple Yakushi-ji de Nara ${ }^{8}$. La plupart des spécialistes s'accordent pour dire qu'il n'y a pas eu " transfert » des bâtiments eux-mêmes, mais la question de savoir si le honzon de Yakushi-ji vient de Moto Yakushi-ji fait débat. Si la triade de Yakushi-ji a été exécutée suite au transfert du temple, elle remonte aux environs de 725, tandis que la triade de Moto Yakushi-ji remonte aux environs de 690. Le laps de temps entre ces deux dates est très bref, mais, au-delà du transfert de la capitale aux environs de 710, les luttes politiques de toute la deuxième moitié du viI siècle, dont l'enjeu était à la fois de transposer et de transcender le système impérial de style chinois, aboutissent alors. C'est du temps de l'empereur Tenmu que se sont développées la terminologie et la symbolique cosmologique du souverain et de la cour importées du modèle chinois, de pair avec les codes de lois, les institutions et la littérature, narrative et poétique. C'est aussi à cette époque qu'apparaissent les premières occurrences des mots " empereur " (tennō 天皇_) et "Japon" (nihon 日本). Je formule ici l'hypothèse que Tenmu Tennō et la princesse consort, qui a pris sa succession sous le nom de Jitō Tennō 持統天皇 (645-703；r. 690-697), occupent une place tout aussi éminente dans le cosmoscape du piédestal que dans les œuvres historiques les plus anciennes, relatives à la mise en place d'un système impérial de style chinois, comme le Nihon shoki de 720 , dont les trois derniers chapitres leur sont intégralement consacrés.

Le piédestal de Yakushi-ji présente un décor unique, dont les racines, des points de vue stylistique et iconographique, remontent dans toutes les parties de l'Asie, notamment en Perse, et même jusqu'à Rome. Ce curieux mélange de motifs n'a jamais reçu d'interprétation satisfaisante, que ce soit en rapport avec la triade du Bouddha de la Médecine, avec les mécènes impériaux du temple, ou avec l'évolution du bouddhisme au cours de son histoire, puisque, comme l'ont montré des recherches récentes, il a intégré plusieurs formes de religion populaire chinoise, dont des éléments de ce qui serait plus tard appelé taoïsme, yin-yang, ou encore tantrisme'. Les analyses sur l'iconographie cryptique du piédestal, que ce soit sur des motifs isolés ou sur le programme intégral, occultent aussi les liens pourtant évidents entre cet objet culturel et d'autres. Les spécialistes japonais présentent souvent le piédestal comme un pastiche :

Je ne peux que supposer que les motifs du piédestal ne viennent pas de la même source mais que des éléments stylistiques provenant de la Chine ancienne ont été sélectionnés et reproduits. Comme il y a par ailleurs des motifs rarement attestés sur un piédestal de Bouddha (les entrelacs de vignes et les quatre dieux animaux shijin 四神), des motifs incompréhensibles dans une perspective iconographique (les douze divinités monstrueuses, jūni no onigami 十二の鬼神), et des motifs adaptés au style japonais (la tortue-serpent noire), nous ne pouvons faire autrement que de conclure à un défaut dans l'expression, malgré une conception impressionnante. La question de savoir s'il faut associer ces caractéristiques au règne de l'impératrice Jitō ou à une époque ultérieure au transfert de la capitale à Heijō [Nara] reste encore à débattre ${ }^{10}$.

Je formule ici l'hypothèse que, loin d'être un pastiche, ce piédestal réunit des figures et des motifs qui représentent autant de signes et de symboles exprimant une vision cosmologique du monde. Je désignerai cette représentation par le terme cosmoscape, un imaginaire à cheval entre iconographie et idéologie visant à donner une vision explicative 
du « monde " (cosmo) représentée (scape, "scène, paysage ») sous la forme d'un ensemble plus ou moins unifié. Cette hypothèse ne revient pas à dire que ces représentations soient " intentionnelles ", car nous ne pouvons pas assimiler les auteurs à leurs créations et nous savons que l'intentionnalité est un terrain glissant. En tout état de cause, la question de savoir si l'icône et le piédestal datent de la période de Moto Yakushi-ji (fin du VII siècle) ou ont été exécutés à Nara au début du VIII ${ }^{\mathrm{e}}$ siècle est de ce point de vue déterminante.

L'imaginaire augural, apotropaïque et magique du piédestal développe un cosmoscape représentatif de ce tournant dans l'histoire du Japon, et, de pair avec l'icône qui le surmonte, il évoque à la fois la loi juste du souverain et l'omnipotence de Bouddha. Moulé, de façon particulièrement appropriée, par paliers, à l'image du mont Meru, montagne cosmique, spirituelle et politique, il représente la conversion et la suppression du mal et des menaces extérieures, le lien entre empereur et sujets, et les idées de vœux propitiatoires et de pouvoirs talismaniques, le tout dans un mélange d'éléments narratifs et symboliques. Le piédestal, qui témoigne de la circulation des symboles et des idées entre les régions, est ainsi à mi-chemin entre cosmoscape et ideoscape ${ }^{11}$ : il est composé de couches différentes reflétant le pouvoir et la vertu de Bouddha et des empereurs, ainsi que leurs rapports avec des puissances et des peuples assujettis et convertis. Exécuté à la demande des héritiers du pouvoir dans la province de Yamato 大和 pour accompagner le Bouddha de la Médecine et les bodhisattva, il met en scène, intentionnellement ou non, le " fondateur impérial " de Yakushi-ji en tant que modèle d'un gouvernement de style chinois dans un cadre cosmologique à la fois bouddhique et sinique.

Les spécialistes japonais ont majoritairement cherché à associer les créatures, les figures, et les motifs hybrides représentés sur le piédestal à des sources spécifiques dans la culture visuelle chinoise ou coréenne ou à des archétypes ethniques des peuples $\mathrm{d}^{\prime}$ Asie du Sud-Est ${ }^{12}$, ou à les étudier de façon à dater le piédestal et/ou la triade sur la base de critères stylistiques, notamment par le biais du motif de la vigne ${ }^{13}$. Ils n'ont pas cherché à resituer les curiosités visuelles du piédestal dans la perspective d'un imaginaire relatif à la formation d'un État impérial de style chinois. Seul Nagaoka Ryūsaku 長岡 龍作 a formulé l'hypothèse que les gouverneurs de Yamato, mécènes de Yakushi-ji au VIII ${ }^{\mathrm{e}}$ siècle, avaient voulu montrer leur sinicisation ${ }^{14}$. Il s'est toutefois concentré sur l'ère Tenpyō 天平 (729-749) et sur l'évolution des "représentations de Bouddha " (butsuzōkan 仏像観) qui l'ont caractérisée, sans souligner l'importance de Tenmu Tennō et de Jitō Tennō du point de vue des origines de Yakushi-ji. Il n'a pas non plus défini le processus de "sinicisation " dans un contexte plus large, notamment en cherchant des analogues au piédestal en matière de construction historique réinterprétant les événements dans la perspective d'un imaginaire impérial.

Selon Herman Ooms, "Tenmu a paré son règne d'une aura surnaturelle en puisant dans un certain nombre de symboles taoïstes ${ }^{15}$ ". Sur la base des figures et des motifs représentés sur le piédestal, je suggère qu'indépendamment de sa date, il représente un cosmoscape relatif à la transformation de la cour de Yamato en un État impérial de style chinois. Avant même les récentes analyses métallurgiques (dont je ferai état plus loin) qui ont associé le piédestal à Moto Yakushi-ji, j'avais formulé l'hypothèse que, s'il n'était pas une copie de celui de Fujiwarakyō et qu'il appartenait à la période Nara, il lui rendait au moins hommage dans sa tonalité ${ }^{16}$. Il témoigne ainsi de la même stratégie iconographique concernant l'histoire de l'empire que les œuvres qui ont établi son autorité politique et culturelle au début du VIII e siècle, comme le Nihon shoki et le Kojiki, par la représentation d'une lignée de gouverneurs de Yamato ayant abouti à Tenmu et à Jitō. L'impasse de la critique japonaise sur l'importance de la triade et du piédestal quant aux liens entre Yakushi-ji (et Moto Yakushi-ji) et ses (ou leurs) fondateurs dans la longue durée tient 
en majeure partie à la réticence des spécialistes japonais, depuis la période Meiji, à douter de la véracité de ces " fondements » de la culture du pays sur le plan historique et littéraire, et à reconnaître que l'histoire de l'empire est le résultat d'une compilation et d'une (ré)interprétation orchestrée par la cour de Yamato au viII siècle, avec la mise en place du système impérial et l'essor du clan Yamato, dans le but de s'approprier les idées des clans immigrés ${ }^{17}$.

Les études du $\mathrm{XX}^{\mathrm{e}}$ siècle sur l'art bouddhique de la période Nara sont imprégnées à la fois de cette vision romancée de l'histoire de l'empire et des volubiles éloges de certains nostalgiques de l'ère Meiji comme Watsuji Tetsurō dans Koji junrei 古寺巡礼 ([Pèlerinages des vieux temples, 1919] 2012) ${ }^{18}$. L'aura de la période Nara explique encore aujourd'hui l'accent mis sur le mécénat impérial ainsi que sur le projet de Bouddha Roshana 東大寺盧舎那仏 de Tōdai-ji 東大寺 (plus connu sous le nom de Daibutsu 大仏 ou Grand Bouddha), tous deux considérés comme liés à l'histoire de l'empire au contraire de toutes les autres pratiques religieuses (comme celles des ascètes des montagnes), du recours à la violence, ou encore du mécénat bouddhique ${ }^{19}$. L'ouvrage bien connu de Torquil Duthie sur le Man'yōshū en parle comme d'un " répertoire varié d'imaginaires impériaux " ayant joué un rôle crucial dans les représentations de la cour de Yamato en tant que " cour impériale ${ }^{20}$ ". Les conclusions sur la date et sur l'origine de la triade de Yakushi-ji ont ainsi été tenues à l'abri de certaines critiques en raison d'une confiance excessive dans le Nihon shoki. Même si l'on reconnaît l'influence de ce texte et de celle que les idéologues nationalistes actifs avant la guerre du Pacifique exercent encore aujourd'hui sur la définition même de la notion d' " empereur » (tennō 天皇; 《 souverain céleste " $)^{21}$ en tant que chef d'un État impérial de style chinois, l'histoire du Japon impérial demeure liée à ce texte. C'est ainsi que se tisse un lien entre passé et présent, notamment dans les études sur Yakushi-ji.

\section{Sous le Bouddha : piédestaux et cosmoscapes}

Le piédestal richement orné, d'une hauteur de $152 \mathrm{~cm}$, est composé d'une première pile de trois sections parallélépipédiques (kamachi 框) reposant sur un socle (kiban 基盤) " en pétale à l'envers " (kaeribana 返花 327,5 × 256,3 cm), montant par paliers, qui supporte la section médiane, ou " taille " (yōbu 腰部), à la fois la plus resserrée et la plus haute $(197,9 \times 52 / 51,5 \mathrm{~cm})$, le tout surmonté par deux sections qui forment des plateaux descendants. Chacun des cinq " paliers » ou " plateaux » est orné de motifs en bas-relief sur son pourtour. Cette forme de piédestal en double pyramide est attestée dans toute la période prémoderne en Chine et en Corée, comme dans la plupart de l'Asie, où elle est symboliquement assimilée à la montagne sacrée, le mont Meru (jp. Shumisen 須弥山), lieu de résidence des dieux au centre d'un univers à la fois physique et cosmologique ${ }^{22}$. Dans la cosmologie bouddhique, le mont Meru est l'axis mundi : s'élevant de l'océan depuis les profondeurs de la terre, il est entouré de sept mers concentriques traversées de continents et de montagnes; le soleil et la lune tournent autour de lui ; les enfers se trouvent au-dessous et, au-dessus, les cieux. Le mont Meru se présente lui-même sous la forme d'une série de paliers au sommet desquels résident les Lokapala, les quatre rois célestes, gardiens des directions (Shitennō 四天王). Beaucoup de spécialistes de l'art japonais seront encore surpris d'apprendre que des icônes bouddhiques bien connues de la période Asuka reposent sur un piédestal ou un socle " en mont Meru " (shumiza 須弥座, shumidan 須弥壇) orné de motifs "non-bouddhiques ", un sujet qui n'est exploré que depuis peu, tout particulièrement par Nagaoka Ryūsaku²3. 
On trouve des piédestaux en mont Meru à Yakushi-ji, mais aussi à Hōryū-ji, dans le kondō sous le Bouddha de la Triade de Shaka (Shaka sanzon zō 法隆寺釈迦三尊像 ; 623) comme dans la salle du trésor sous le Tamamushi no zushi (Tamamushi zushi 玉虫 㕑子). Considéré par certains spécialistes comme une ouvre réalisée dans la péninsule Coréenne, ce dernier se dresse sur son socle comme un "palais miniature ${ }^{24}$ " (gūdenzō 宮殿像, nom sous lequel il est désigné dans un document de 747, zushi n'étant qu'une appellation moderne) figurant le royaume de Bouddha, dont les panneaux sont saturés de peintures des quatre rois célestes et de deux bodhisattva. Le panneau du bas à l'arrière représente le mont Meru sur les trois quarts supérieurs et un palais du Dragon sous-marin tiré du Sütra du roi-dragon dans le quart inférieur ${ }^{25}$. En plus du mont Meru et de la mer, on trouve des rois célestes en lévitation, le soleil et la lune, ainsi que des immortels et d'autres symboles de la religion populaire chinoise, dont beaucoup sont issues du taoïsme. Les autres panneaux montrent des scènes tirées de textes bouddhiques.

Pour ce qui est de la Triade de Shaka, le piédestal du Bouddha est constitué de deux blocs de bois empilés (un style appelé nijūsenjiza 二重宣字座), peints de motifs qui n'ont pas de connotation bouddhique (du moins pour la plupart, car, sur ce point, les opinions varient) mais qui sont inspirés de l'iconographie du mont Meru et du royaume chinois des immortels : ainsi trouve-t-on des pics, de larges arbres, les vagues de la mer sous le mont Meru, les quatre rois célestes résidant à son sommet, des figures qui semblent être des ascètes ou des hommes en méditation, et des êtres célestes ${ }^{26}$. Ce décor, comme celui du Tamamushi no zushi, nous conduit à remettre en question les déclarations sur l'art bouddhique antérieur à la période de Nara et notamment à reconnaître le rôle important des croyances non-bouddhiques dans le symbolisme des icônes bouddhiques. L'iconographie du mont Meru dans l'art bouddhique est encore mal comprise, et ce n'est que depuis peu que des photographies infrarouges des piédestaux des Bouddhas de Hōryū-ji et de Yakushi-ji nous en offrent des représentations lisibles ${ }^{27}$.

Il est fondamental de situer le piédestal du Bouddha de Yakushi-ji dans ce contexte plus large. Il apparaît ainsi comme le support d'une représentation cosmologique, comme un cosmoscape " sous le Bouddha " dans la tradition des piédestaux en mont Meru instituée au VII ${ }^{\mathrm{e}}$ siècle. Il est, de fait, le dernier représentant connu de cette " lignée " d'œuvres, même si la littérature académique ne le présente pas ainsi. Le mélange qui le caractérise cesse aussi d'apparaître comme une anomalie ; tout comme ses pairs de Hōryū-ji, il puise dans une iconographie contemporaine, liée à des croyances, à une médecine, à des connaissances rituelles, à des textes, des idées et des pratiques à la fois bouddhiques et "non-bouddhiques " (selon la dichotomie traditionnelle de la littérature académique). Comme je vais maintenant le démontrer, le piédestal du Bouddha de Yakushi-ji opère un glissement dans une iconographie déjà représentée sur d'autres piédestaux, liée au royaume chinois des immortels et des esprits, au gouvernement juste et à la conversion du démoniaque ou de l'étranger. En tant que " piédestal ", il est à mi-chemin entre deux monstres sacrés de l'histoire de l'art bouddhique japonais, le piédestal de la Triade de Shaka de Hōryū-ji décrite plus haut et le piédestal du Grand Bouddha de Tōdai-ji, réalisé 125 ans plus tard : immense, très différent, avec ses vingt-huit pétales de lotus et sa représentation du mont Meru sur l'un d'entre eux, il reflète des concepts dérivant du Kegonkyō 華厳経 [Sūtra de l'ornementation fleurie] et du Bommōkyō 梵網経 [Sūtra du filet de Brahma] ${ }^{28}$. Les liens conceptuels entre ces piédestaux ont été négligés. Les spécialistes opposent motifs bouddhiques et "non-bouddhiques " alors que, comme ces objets le démontrent, le bouddhisme inclut ces éléments conceptuels et iconographiques. 
Il est essentiel pour cette étude de rappeler que les empereurs antérieurs à Tenmu avaient déjà exploité la religion populaire chinoise dans des sources littéraires et artistiques et déployé une large gamme de pratiques rituelles et religieuses, que ce soit à titre personnel ou sur un mode collectif à la cour. La quatrième année du règne de Tenmu (675), les étudiants de plusieurs départements du ministère des Affaires centrales, dont celui de la Divination (Onmyōryō 陰陽寮), « ont déposé des offrandes de remèdes et de trésors rares, tout comme des personnes venues d'Inde, de Bactriane, de Baekje et de Silla. Ce même jour, l'empereur a pris des boissons médicinales ${ }^{29}$... " La citation suivante de Livia Kohn sur les " sources » chinoises est aussi hautement pertinente pour le Japon des périodes Asuka et Nara :

L'interaction complexe entre taoïsme et bouddhisme dans la Chine médiévale est souvent résumée par le qualificatif de "bouddho-taoïste". Sous les dynasties Sui et Tang, ces deux religions ont fini par servir les ambitions impériales et par déployer de vastes réseaux de temples dans tout le pays. Les relations les plus étroites entre les deux sont à chercher dans les domaines de la philosophie et de la cosmologie. Elles ont conduit à l'émergence de nouvelles formes et de nouveaux procédés du point de vue des pratiques religieuses, avec un jeu d'influences mutuelles. Il ne s'agit pas là à strictement parler de "syncrétisme", terme qui implique que "deux religions, cosmologies, rituels ou pratiques jusque-là distincts forment un mélange et qu'un changement de circonstances les séparerait naturellement ${ }^{30 "}$.

Au cours de son règne, Tenmu a fondé plusieurs départements ministériels comme ceux de la Divination et des Divinités (Kami, Kamitsukasa ou Jinkan 神官, ultérieurement Jingikan 神祇官), avec notamment un maître du Yin et du Yang, et il a cherché des modes de protection et de guérison dans le bouddhisme (il a ordonné la copie intégrale du canon bouddhique [Tripitaka] en 673.3) mais aussi, grâce à des intermédiaires, dans des pratiques issues de la péninsule Coréenne. Ces éléments glanés dans le Nihon shoki peuvent nous permettre de mieux comprendre les enjeux de la représentation du piédestal, même si elle constitue, au même titre que toute forme de représentation ou de récit, une fiction.

\section{Deux capitales, deux temples et deux icônes}

L'impératrice consort tomba malade. Peu après, l'empereur [Tenmu] prononça un vœu pour son rétablissement et fonda [Moto] Yakushi-ji. [Il] contraignit aussi cent hommes à renoncer au monde. À la suite de quoi, elle retrouva la santés1.

La date d'achèvement de Moto Yakushi-ji est inconnue, mais des éléments archéologiques comme l'analyse des tuiles (kawara 瓦) et les témoignages relatifs à des événements rituels à [Moto] Yakushi-ji et dans d'autres monastères de la région d'Asuka conduisent les spécialistes à conclure que les bâtiments essentiels du temple étaient achevés au début de 688, et l'ensemble du complexe en 698. Le Nihon shoki évoque, en 688.1.8, un rituel correspondant au deuxième anniversaire de la mort de Tenmu Tennō ${ }^{32}$. Étant donné que les bâtiments essentiels du temple (pavillon principal, pavillon des prières, pagodes, dortoirs des moines) et l'icône principale doivent être achevés avant qu'un tel rite ne puisse être effectué, je me range du côté de ceux qui, comme Mizuno Keizaburō 水野敬三郎, Ōhashi Katsuaki 大橋一章, Naitō Sakae 内藤栄, et d'autres, pensent que le kondō et le honzon étaient sans doute achevés en 688, huit ans après le vœu de Tenmu ${ }^{33}$.

Comme Yakushi-ji le serait plus tard, Moto Yakushi-ji hébergeait un Bouddha de la Médecine jōroku assis en bronze coulé, orné d'une mandorle avec sept Bouddha et monté 
sur un piédestal en mont Meru avec ses deux bodhisattva, Nikkō et Gakkō, à ses côtés. La triade de Nara a-t-elle été transférée telle quelle de Fujiwarakyō ? Et qu'en est-il des bâtiments, sachant que les idéogrammes chinois utilisés dans les textes prémodernes pour exprimer l'idée de “transfert/déplacement " (移, 移動, 移伽, 徙建 et 移建, avec toutes leurs variantes $)^{34}$ peuvent avoir un sens littéral autant que figuré ? Le débat académique a essentiellement porté sur ces questions pendant des décennies. Les similarités mises en évidence par les fouilles de Moto Yakushi-ji par rapport à la disposition des bâtiments essentiels de Yakushi-ji ont d'abord conduit les spécialistes à conclure que certains des bâtiments originaux de Fujiwarakyō avaient été déplacés à Nara. Je suis plutôt d'avis que le plan original de Yakushi-ji a été inspiré de celui de Moto Yakushi-ji dans un élan global de commémoration de l'original.

Les rapports archéologiques de 1987 et les recherches de Hanatani sur les traces d'implantation des poteaux et sur la composition des tuiles ont démontré de manière convaincante aux yeux de nombreux chercheurs que les bâtiments de Yakushi-ji ont été érigés de toutes pièces et non déplacés ${ }^{35}$. Lors du "Colloque sur Yakushi-ji de Heijō [Nara] : réflexions sur le sens du "transfert" du temple " au Musée national de Nara en 2019, plusieurs spécialistes ont présenté des éléments concordants montrant que le " transfert " avait été figuré, et non littéral ${ }^{36}$. Du point de vue de l'archéologie, et en particulier de l'analyse des tuiles, Ishida Yukiko 石田由紀子, de l'Institut du patrimoine culturel de Nara, a reconstitué la chronologie suivante sur la base d'une comparaison des motifs et de la distribution des tuiles sur le site de Moto Yakushi-ji : pavillon principal (kondō), porte intérieure (nakamon 中門), pagode de l'Est (tōtō 東塔), et enfin pagode de l'Ouest (saitō 西塔) ${ }^{37}$. À mon avis, le kondō et le honzon de Moto Yakushi-ji ont été achevés entre 688 et 692, et la construction s'est sans doute poursuivie sous le successeur de Jitō Tennō, son petit-fils Monmu Tennō 文武天皇 (683-707; r. 697-707) ${ }^{38}$.

Les partisans de l'idée que la triade de Yakushi-ji est du VII ${ }^{\mathrm{e}}$ siècle et qu'elle a été transférée de Fujiwarakyō à Nara citent à l'appui plusieurs témoignages sur un autre transfert de Fujiwarakyō à Nara : celui du honzon de Daikandai-ji, l'idée d'un transfert physique du célèbre Bouddha Saākyamuni de Daikandai-ji (Shaka Nyorai 釈迦如来), aujourd'hui détruit, étant communément acceptée ${ }^{39}$. On ne trouve cependant pas de témoignages de ce genre sur le honzon de Moto Yakushi-ji. Dans un article de 2015, le directeur et conservateur en chef du Musée national de Nara, Naitō Sakae, a rouvert le débat en mettant au dossier un document qu'il croyait daté du VIII ${ }^{e}$ siècle (période de Tenpyō), selon lequel le honzon de Nara, son piédestal, sa mandorle et ses deux bodhisattva, " avaient été pieusement façonnés et installés par Jitō Tennō $\overline{0}^{40}$ " (autrement dit, provenaient de Fujiwarakyō). Ce document est cependant considéré par la plupart des spécialistes comme un faux datant du XI ${ }^{\mathrm{e}}$ siècle, ne constituant pas, à ce titre, une preuve valable.

\section{Le piédestal du Bouddha de Yakushi-ji : matériaux et motifs}

Les spécialistes ont toujours cru que le Bouddha de Yakushi-ji et son piédestal avaient été coulés à la même époque et dans le même alliage. Si aujourd'hui, le honzon est daté, sur le site internet de Yakushi-ji comme dans la littérature spécialisée, de la période Hakuhō à la fin du VII ${ }^{\mathrm{e}}$ siècle, ce qui revient à en faire l'icône de Moto Yakushi-ji, les recherches surprenantes et encore inédites présentées par Fujioka au colloque du Musée national de Nara en 2019 ont démontré de manière convaincante, sur la base d'une double comparaison entre compositions métallurgiques et motifs stylistiques, que la triade composée par le Bouddha de la Médecine et ses deux bodhisattva, Nikkō 


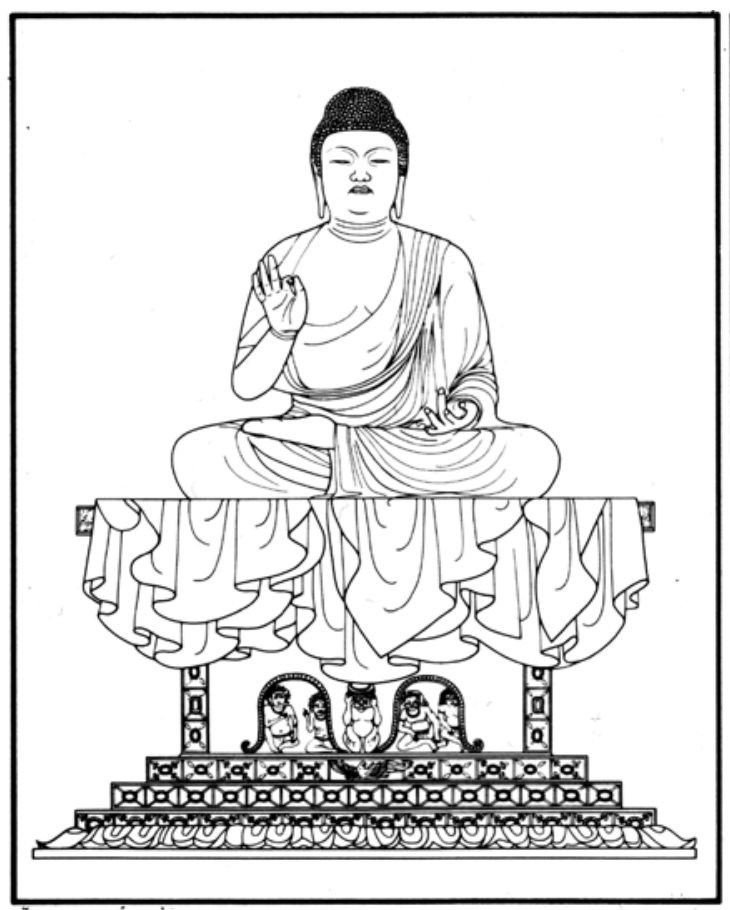

67. 町田説による本尊並び台座の復原図

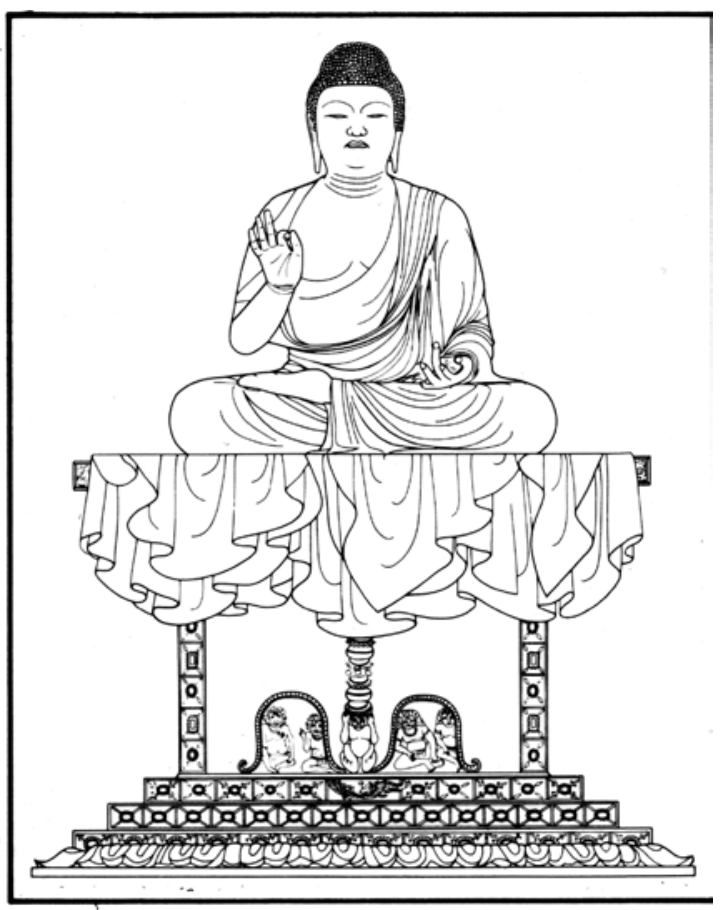

66. 田村説による本尊並び台座の復原図

et Gakkō, a très probablement été réalisée au cours de la période Nara (soit après 710). Si ce résultat allait dans le sens de l'opinion majoritaire, la surprise est venue du piédestal, qui pourrait avoir été coulé antérieurement, en l'occurrence avant le transfert à Nara ${ }^{41}$.

3. Dessins de Machida Kōichi (à gauche) et de Tamura Yoshinaga (à droite) représentant la reconstitution du piédestal de Moto Yakushi-ji avant sa réduction. Issu de Machida, 1984, cité n. 43, p. 117, fig. 66 et 67.

Sur la base des impuretés et du pourcentage de cuivre utilisé pour le bronze des statues, le Bouddha peut être daté de la période Nara (après le transfert), tandis que le piédestal présente un alliage similaire à des œuvres antérieures (avant le transfert), avec en l'occurrence un moindre degré de cuivre $(93,8 \%)$ que le Bouddha qui le surmonte $(97 \% \text {, soit un taux très élevé })^{42}$. D'autres critères, en particulier stylistiques, permettent aussi généralement d'associer le Bouddha à d'autres œuvres de la période Nara faites de matériaux similaires. Comment expliquer que le piédestal ait pu être transféré sans son Bouddha d'origine, si c'est ce que suggèrent ces nouveaux éléments ? Comment les deux ont-ils pu être séparés ? Les spécialistes jugent souvent que le piédestal a été moulé en trois phases : la section inférieure avec le socle et les trois paliers du bas, la section médiane ou " taille ", et les deux plateaux supérieurs. Ils ignorent souvent que la section médiane a été tronquée dans sa partie haute à une certaine époque, pour des raisons inconnues, le piédestal d'aujourd'hui étant ainsi moins élevé que l'original. On peut en voir des traces sur les reliefs de la face nord (fig. 2) en observant les cellules ornées de motifs lotiformes en haut des lisérés verticaux. Il est difficile d'évaluer l'ampleur de cette réduction : a-t-on coupé seulement la portion de cellule manquante, ou bien plusieurs cellules au-dessus ? On trouve dans une monographie de 1984 deux planches de reconstitution du piédestal (fig. 3) opposant les hypothèses de Machida Kōichi 町田甲一 (à gauche) et de Tamura Yoshinaga 田村吉永 (à droite $)^{43}$. Sur la question de savoir pourquoi le piédestal a été coupé, Machida suggère, 


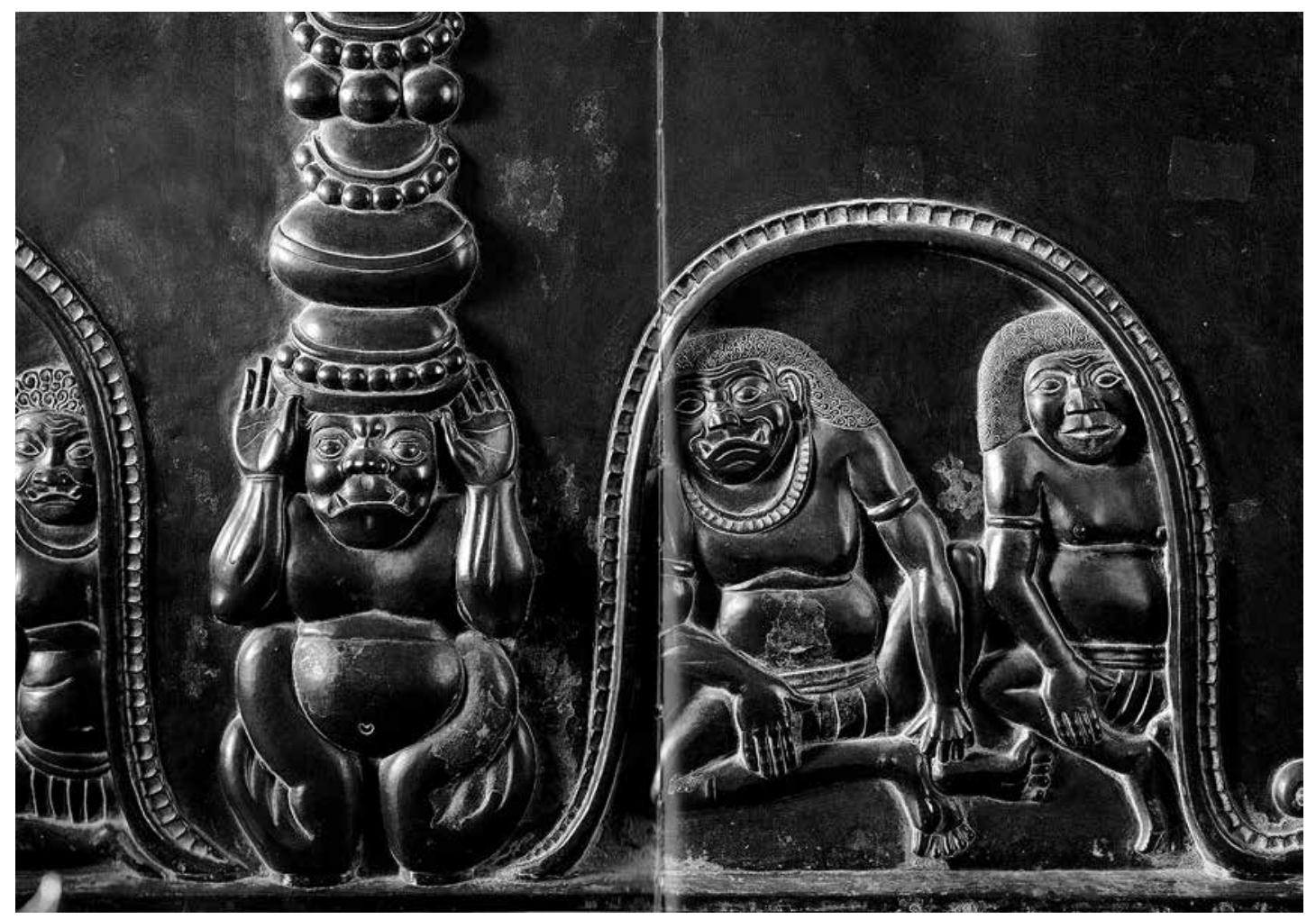

4. Piédestal du Bouddha de Yakushi-ji, face nord (arrière), détail des yaksha et de la créature hybride. Issu de Nishimura Kōchō, Nishikawa Kyōtarō et al., Yakushi Sanzon: Nara Yakushiji Kondō. Medaka no hon. Miwaku no butsuzō, Tōkyō, Mainichi shinbunsha, 2001, n.p. comme d'autres, une faiblesse due à la présence de bulles d'air intervenues dans le processus de coulage ${ }^{44}$. Les recherches métallurgiques de Fujioka soulèvent encore beaucoup de questions intrigantes, parmi lesquelles celle de savoir ce qu'il est advenu du Bouddha de Moto Yakushi-ji dépourvu de son piédestal. Si de telles questions ne peuvent être ici qu'évoquées, il apparaît crucial de signaler, dans le débat sur l'iconographie du piédestal, qu'il est sans doute plus adéquat de le faire remonter au VII ${ }^{e}$ siècle, et que la réduction de sa partie médiane a altéré la lisibilité de son programme (je pense en particulier au motif de la créature hybride des faces nord et sud, avec un pilier sur la tête soutenant le Bouddha, qui, spatialement, peut paraître incongru), sans oublier qu'une hauteur plus importante conférerait à l'ensemble un meilleur équilibre visuel.

Les analyses métallurgiques suggérant que le piédestal date de Moto Yakushi-ji concordent fondamentalement avec mon hypothèse qui consiste à le considérer comme une copie du piédestal de Moto Yakushi-ji - car, comme je vais le démontrer, les motifs sont bien ancrés dans l'époque de Tenmu et Jitō en termes de style, d'histoire iconographique, et de lien idéologique. Il reste pourtant difficile de faire la différence entre l'idéologie impériale de l'époque de Tenmu et celle qui est apparue après le transfert de la capitale à Nara, et le piédestal n'offre sans doute pas un reflet de toutes les luttes politiques évoquées dans les versions du Nihon shoki, si du moins celles-ci sont fiables. Si le piédestal a été réalisé à Nara, il l'a été dans le même esprit commémoratif que les éléments découverts dans la pagode de l'Est du VIII ${ }^{\mathrm{e}}$ siècle, qui, commencée en 730.3.9, a survécu ${ }^{45}$. Une inscription 
au niveau de la flèche (sōrin 相輪) énonce les éléments clés dans l'histoire de ce temple : les deux empereurs responsables de son transfert, et son rôle dans la vie religieuse de la nouvelle capitale $^{46}$. Il me semble également possible que les bâtiments principaux de Yakushi-ji à Nara aient été disposés comme ceux de Moto Yakushiji, malgré les dimensions bien plus importantes du nouveau temple ${ }^{47}$. À travers la reconstruction du temple comme à travers l'art et la littérature, Yakushi-ji offre ainsi l'exemple d'un processus historique dans lequel l'empereur Tenmu et l'impératrice Jitō ont été célébrés comme les héritiers naturels de l'ordre impérial.

Les motifs lotiformes présents un peu partout sur le piédestal du Bouddha rappellent les motifs

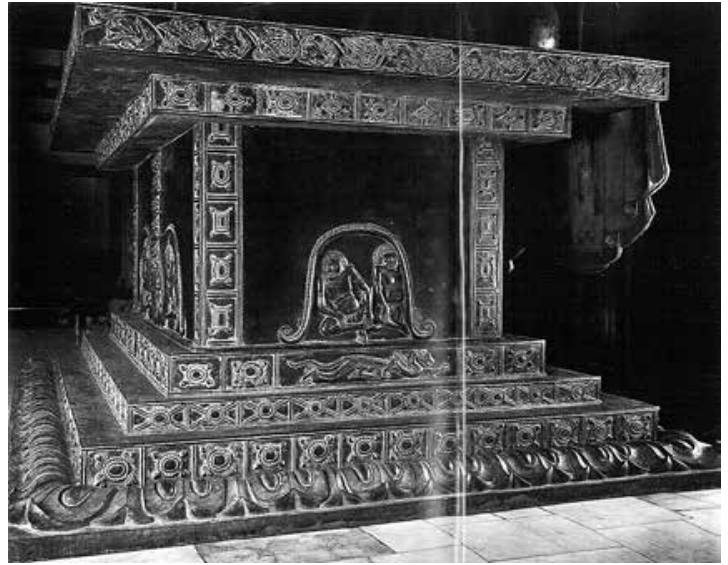

5. Piédestal du Yakushi Nyorai, face ouest. Issu de Nishimura, Nishikawa, 2001, n.p. de couronnes et de bijoux des deux bodhisattva du honzon de Yakushi-ji, datés du viII e siècle, mais aussi des icônes de la période de Hakuhō. Les motifs en $\mathrm{X}$ qui leur servent de fond sur le deuxième palier du bas évoquent quant à eux un motif utilisé sur des pièces wushu du type de celles retrouvées dans la tombe du roi baekje Muryeong (462-523; r. 501-523) à Gongju ${ }^{48}$ (les pièces wushu étaient déposées dans les tombes).

Les reliefs les plus frappants de la section centrale du piédestal sont les douze personnages habillés de pagnes dans les niches ourlées, tous présentés par couples : un couple sur chacune des "petites " faces est et ouest, deux couples sur chacune des " grandes " faces nord (fig. 4 et 5) et sud. En termes d'iconographie bouddhique, ils représentent très probablement les douze généraux célestes (Jūnijinsho 十二神将), qui, dans les sūtra sur le Bouddha de la Médecine, exaucent les douze vœux de celui qui est encore Bodhisattva. Ces douze vœux sont énumérés dans la première section du Sūtra sur les voux et les mérites originaux du Maître de Médecine Tathagata Lumière de Lapis Lazuli, qui était sans doute connu dans la traduction de Xuanzang 玄牀, datée des environs de $650^{49}$. Très divers - atteindre le parfait éveil ; illuminer les esprits ; être nourri, vêtu, et paré ; rester à l'abri des pensées négatives et des châtiments royaux ; veiller aux préceptes ; guérir les maladies et les limitations physiques, etc. -, ils ne peuvent se résumer à une guérison corporelle $^{50}$. Si les douze généraux célestes étaient jusque-là des yaksha 夜叉 (sk. yakșa), les forces du mal évoquées dans de nombreux textes bouddhiques, ils se révèlent d'une grande bienveillance une fois convertis. Raoul Birnbaum évoque ainsi leur conversion :

À l'époque où Śākyamuni révéla le sūtra sur le Bouddha de la Médecine (Bhaisajya-guru), il y avait douze généraux yaksha dans l'assemblée.

Selon la tradition indienne, les yaksha sont des esprits farouches qui causent souvent des maladies par possession démoniaque. Ces féroces généraux, chacun à la tête de sept mille yaksha, finirent par trouver refuge dans les Trois Trésors et par vouloir aider tous les êtres sensibles. Ils firent en particulier le vœu d'aider ceux qui feraient circuler le Sütra Bhaisajya-guru et qui accepteraient et honoreraient le nom du Bouddha de la Guérison. Avec leurs troupes, ils firent ainsi le vœu de garder et de protéger ces gens, en les libérant de la douleur et de la souffrance et en facilitant leurs pas. Ils prescrivirent aussi un rituel simple pour invoquer le Bouddha de la Guérison en vue de guérir les maladies ${ }^{51}$. 
Il me semble que la référence visuelle au mont Meru ne se limite pas à la forme du piédestal et qu'elle englobe aussi les yaksha de la tradition hindoue, gardiens de Kubera, le premier des quatre rois célestes (Lokapāla), gouverneur du Nord et protecteur des justes. Entre les deux niches ourlées contenant les couples de yaksha sur les faces nord et sud apparaît aussi une créature hybride peut-être inspirée de l'iconographie de Kubera, dotée de canines saillantes et portant sur son crâne un pilier serti. Dans la tradition artistique de l'Asie du Sud (on pense aux torana des stūpa de Sanchi), Kubera et les yaksha sont souvent de dimensions naines et légèrement ventrus, comme c'est le cas ici. Les pieds amphibies de cette créature hybride s'inscrivent peut-être dans la tradition des motifs ondulants, attestés en Asie de l'Est, des jambes et des pieds des démons serviteurs du bouddhisme, que ce soient les "monstres ailés " servant de piliers dans l'architecture chinoise, comme dans les grottes de Xiangtangshan 响堂山石窟 de la dynastie Qi du Nord (550-577) ${ }^{52}$, ou les " tuiles démoniaques " de la péninsule coréenne, avec leurs motifs de rouleaux que l'on retrouve dans le Japon du viII siècle, comme le montrent les onigawara 鬼瓦 ( « tuiles ogres ») du palais Heijō de Nara ${ }^{53}$. La littérature spécialisée n'offre cependant aucune source convaincante, ni pour la créature hybride, ni pour les douze yaksha, même si une découverte n'est pas impossible. Les piliers sertis sont attestés à la fois en Chine et en Asie du Sud et, si l'on garde à l'esprit que le piédestal a été coupé, la composition devient moins arbitraire. En février 2018, en visitant les grottes du mont Maiji 麥積山 dans la province chinoise de Gansu, j'ai remarqué des créatures démoniaques en appui sur d'autres, accroupies, qui portaient les piliers soutenant le dais qui abritait le Bouddha Maitreya. On peut les voir à droite et à gauche du registre inférieur de la stèle 10 (dite " Maitreya ") de la grotte 133 (dite " de la Stèle »), datée de 565, d'une hauteur de 1,60 m (fig. 6).

Le contour ondulant des niches sur la section médiane du piédestal est d'une exécution curieuse et forme un élément à la fois végétal et architectonique, avec ses pointes de fougère et son bord extérieur à motif réticulé. Dans un ouvrage primé, Andō Yoshika y voit la dernière apparition au Japon d'un motif de la période Gupta (milieu du III ${ }^{\mathrm{e}}$ - milieu du V siècle) en Asie du Sud, celui du bourgeon (me 芽) de lotus (hasu 蓮) « en tentacule de pieuvre " (tako ashi moyō 蛸足模様), attesté au Japon sur le seul étang aux lotus de l'autel de Tachibana de Hōryū-ji (VII siècle) : encore un élément iconographique de la période Hakuhō liant le piédestal du Bouddha de Yakushi-ji à Moto Yakushi-ji ${ }^{54}$. Cette courbure particulière, qui reste subaquatique dans la nature, jaillit au-dessus du niveau de l'eau dans la tradition artistique de la période Gupta, où elle symbolise l'abondance, la richesse et la bonne fortune des yaksha par sa forme ourlée qui, dans le piédestal, vient insuffler une force vitale à l'ensemble : ainsi dessine-t-elle un motif végétal ascendant, lié à la naissance et à la démultiplication des yaksha (on pense aux peintures de la grotte 17 $\mathrm{d}^{\prime}$ Ajanta, du V $\mathrm{V}^{\mathrm{e}}$ siècle) ${ }^{55}$. Dans la tradition artistique du Japon ancien, elle ne délimite jamais le contour d'une niche et, s'il s'agit effectivement du même motif, il est bien plus stylisé dans le piédestal.

Je vais maintenant m'attarder sur un aspect extrêmement atypique - et à ce jour entièrement négligé - des douze figures réparties dans les niches et des deux piliers les séparant sur les faces nord et sud, qui marque une ressemblance frappante avec des œuvres anciennes de l'Asie du Sud, comme les piliers de la vedikā du grand stūpa d'Amarāvatī (vers 50) ou le torana sud du stūpa 3 de Sanchi, sur la face avant de son architrave supérieure ( $\mathrm{I}^{\mathrm{er}}$ siècle). La section médiane du piédestal du Bouddha de Yakushi-ji me semble témoigner d'une réinterprétation de la " corde " ondulante du lotus (elle-même

6. Stèle Maitreya (10) de la grotte dite " de la Stèle » (133), dynastie Wei du Nord (386-534), premier quart du $\mathrm{VI}^{\mathrm{e}}$ siècle, pierre, $106 \times 80 \mathrm{~cm}$, Chine, grottes du mont Maiji 麥積山, province de Gansu. 


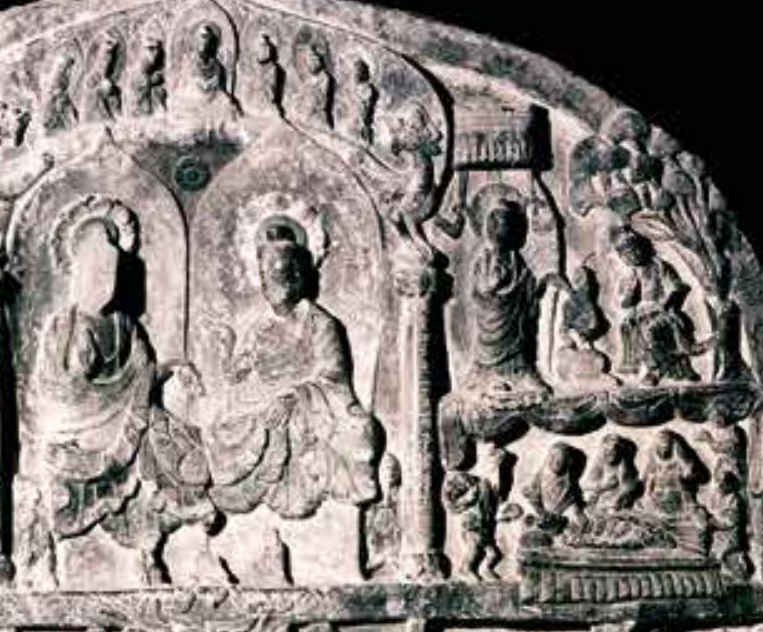

(ivi

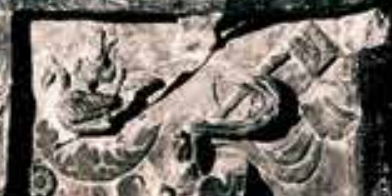

(3)

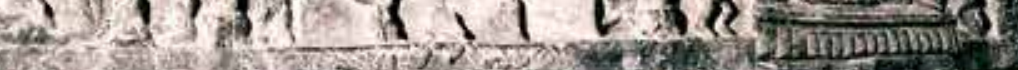

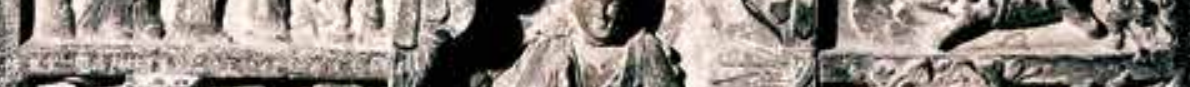

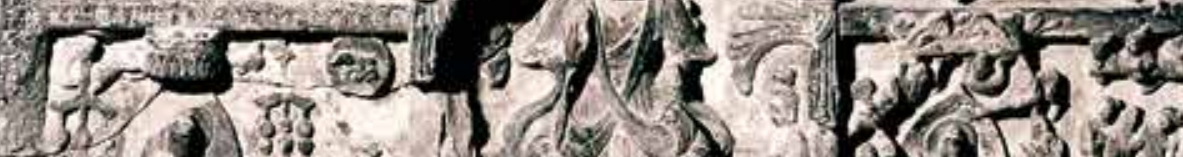

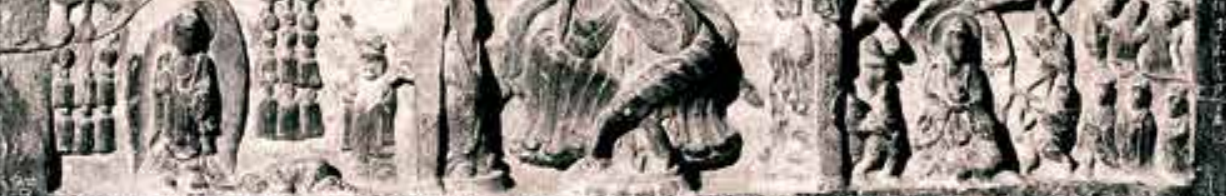

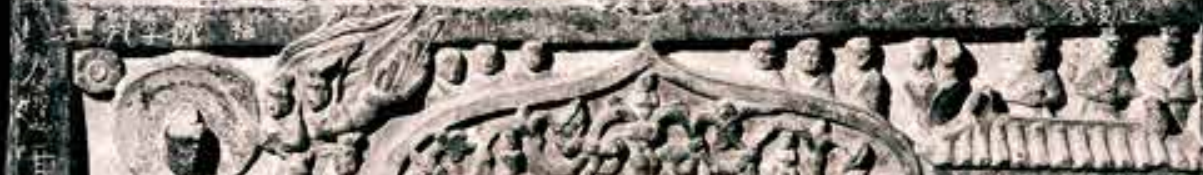

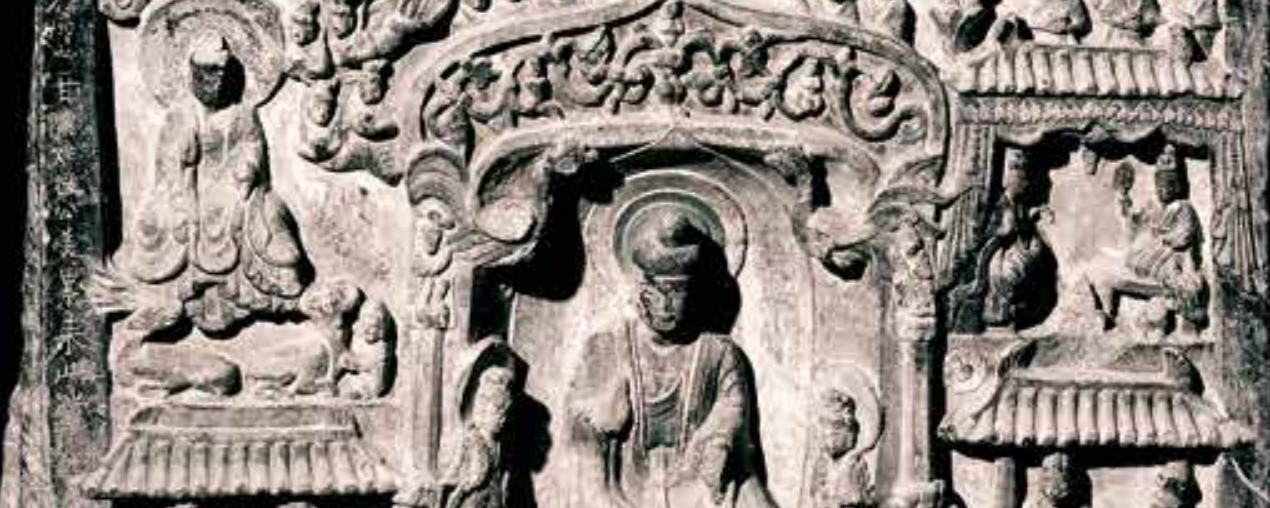

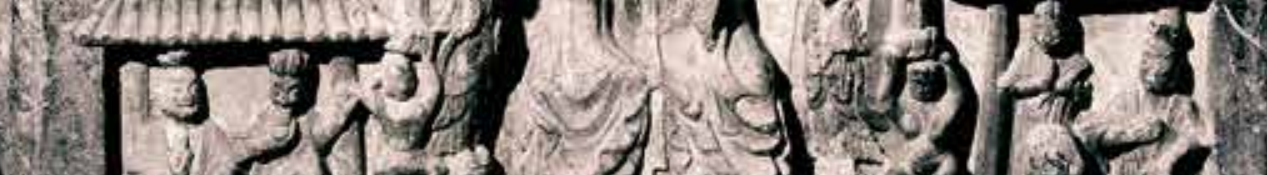

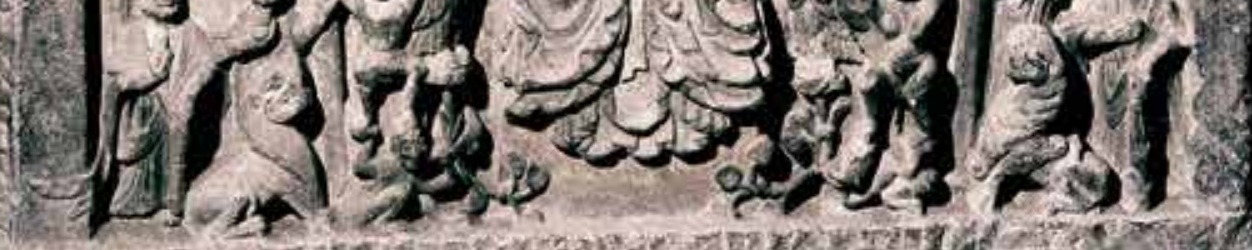

2.5 


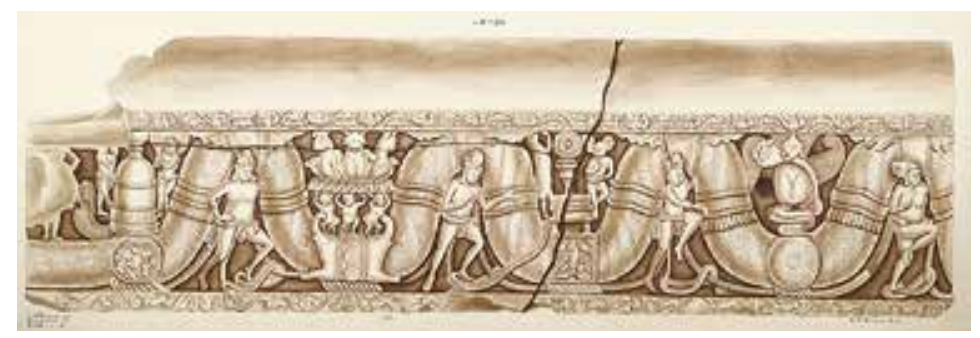

7. Murugesa Moodaliar, copie du chaperon d'un pilier sculpté dans le grand stūpa d'Amarāvatī (détail), vers 1853, lavis sur papier, Londres, The British Library, WD2242-2283, 2256 dérivée de la palmette dans l'art roman), où elle est désormais soutenue par un yaksha aux dimensions naines. Au XIX ${ }^{e}$ siècle, Murugesa Moodaliar a réalisé 42 planches (89 dessins) sur Amarāvatī et sur le Sud de l'Inde, aujourd'hui dans les collections de la British Library. Sur l'un de ces dessins (fig. 7), Yaksha apparaît debout, soutenant une "vigne ondulante ", entouré de créatures naines soutenant elles-mêmes un piédestal à motif de lion : tous ont les bras levés exactement comme la créature hybride du piédestal du Bouddha de Yakushi-ji, porteuse du pilier serti ${ }^{56}$. On assiste, me semble-t-il, à une transposition de la composition globale associant une "vigne ondulante " / " corde ondulante " à Kubera / Yaksha entouré de figures propitiatoires de dimensions naines, une composition attestée au I ${ }^{\text {er }}$ siècle en Asie du Sud avec les ascètes dans les grottes et en Asie centrale avec les immortels chinois dans des paysages montagneux.

Sur le piédestal du Bouddha de Yakushi-ji, les couples de yaksha sont répartis de manière à communiquer avec le "spectateur " ou du moins avec l'extérieur : parfois, un pied déborde de la niche vers le palier juste au-dessous (avec les quatre animaux, ou « dieux des quatre orients " chinois) et, dans tous les cas, les yaksha ont le regard tourné vers l'extérieur (qu'ils regardent devant eux, vers le bas, ou sur le côté). Les poses et les niches évoquent étrangement la grotte du Moulin à prières, à Kizil, près de Baicheng, dans la préfecture d'Aksu (région autonome ouïghoure du Xinjiang, en Chine), remontant à la fin du IV siècle ou au début du $V^{e}$ siècle et témoignant d'une forte influence indienne ${ }^{57}$. Sur le piédestal du Bouddha de Yakushi-ji, les " ascètes " cèdent la place à des figures actives - dont les gestes et les expressions vont de la méditation à l'écoute ou de la pénitence à la grimace, aux lèvres retroussées ou aux canines saillantes - mais jamais menaçantes, ce qui signale qu'elles ont été converties et sont peut-être en train de prêcher l'exemple.

Ces figures paraissent d'abord fantaisistes, mais une étude approfondie de leur aspect physique - leur chevelure plus ou moins bouclée, leur musculature, leurs visages vieux ou jeunes, leur expression, et jusqu'à l'apparition des canines - procède d'une représentation " réaliste " de leur appartenance ethnique, appuyée notamment sur la variation génétique des relations dentofaciales ${ }^{58}$. Comme de nombreux chercheurs, je pense que ces yaksha sont inspirés des descriptions chinoises des périodes Sui, Tang, et ultérieures, présentant les peuples non-Han originaires des îles ainsi que du Sud et du Sud-Est du continent, qui étaient nommés « esclaves de Kunlun » (kunlun nu 崑崙奴), même si le mot " esclaves " ne convient pas tout à fait puisque des maîtres, des mystiques, des traducteurs, et des paysans libres venaient aussi de ce lieu semi-mythique appelé " Kunlun ». La plus ancienne référence attestée à ces " esclaves de Kunlun » se trouve probablement dans le Nanhai jigui Neifazhuan 南海寄歸內法傳 [Tableau de la religion bouddhiste telle qu'elle est pratiquée en Inde et dans l'archipel malais, littéralement "Tableau de la loi intérieure, renvoyée au foyer depuis la mer du Sud », le terme 
de « loi intérieure " exprimant l'idée de " loi cosmique " 内法 $]^{59}$. On ne trouve pas de représentations semblables à celles de notre piédestal dans la tradition artistique de la période Tang, même si les personnes à la peau noire sont attestées dans les statues et dans les peintures ${ }^{60}$. Ces étrangers avaient une présence à la fois réelle et mythique dans les différentes capitales chinoises en relation avec la cour de Tenmu et de Jitō. Les spécialistes s'accordent à penser que kunlun nu désigne les Chams arrivés par voie de mer du royaume de Champā ou, selon Nancy Tingley, " des royaumes côtiers situés entre la province de Binh Thuan dans le Sud et la province de Quang Tri dans le Nord " du Vietnam moderne : leur principale source de revenus était le commerce maritime des produits de leurs terres entre les bassins fluviaux près du littoral et les montagnes ${ }^{61}$. Le Funan était probablement le carrefour culturel le plus important en Asie du Sud et, avec l'essor du commerce et du bouddhisme en Chine et en Asie du Sud-Est, " les Chams et les Funanais construisirent des navires marchands plus grands, inspirés de modèles indiens ", tandis que les prêtres bouddhistes entreprirent le long voyage entre la Chine et l'Inde : ce fut ainsi qu'au VII ${ }^{\text {e }}$ siècle, prêtres et émissaires japonais entendirent parler des Chams et commencèrent à importer depuis le royaume de Champā. Selon Martin Stuart-Fox, "les frontières culturelles [de la Chine] ont été fixées en même temps que la frontière politique entre les Vietnamiens et les Chams ; ou, selon la terminologie chinoise, entre les barbares de l'intérieur et les barbares de l'extérieur "; tous payaient un tribut à la Chine, mais " si les Vietnamiens étaient contraints de vivre sous la domination impériale et d'adopter la culture chinoise, les Chams envoyaient leurs délégations en tant qu'administration indépendante et n'étaient pas sous une pression du même ordre ${ }^{62} "$.

Le Jiu Tangshu 舊唐書 [Histoire des anciens Tang] en donne la description suivante : "Les habitants de Linyi [Champa], vers le sud, ont les cheveux bouclés et la peau noire. Ils sont collectivement appelés kunlun ${ }^{63}$. " Dans un article sur le piédestal du Bouddha de Yakushi-ji, l'historien de l'art Asanuma Takeshi estime que, dans les représentations de la dynastie Tang, les habitants du Sud, les esprits démoniaques ( $a k k i$ 悪鬼) et les humanoïdes anthropophages (rasetsu 羅刹, sk. rāksasa) formaient tous la catégorie globale des kunlun $n u^{64}$. Si les douze yaksha sont dépeints comme des "barbares " du Sud (non-Han), les canines saillantes de certains d'entre eux les désignent selon lui spécifiquement en tant que yaksha, autrement dit comme des figures bouddhiques. Je préfère les envisager dans la perspective d'un cosmoscape global, ne serait-ce que parce que leur représentation vient surmonter celle des quatre animaux. S'ils montrent leurs canines, c'est pour que nous les voyions à la fois comme les représentants d'une minorité ethnique étrangère au « centre " impérial et comme des esprits démoniaques convertis au message bouddhique. Remarquons par ailleurs que la créature hybride porteuse de pilier a aussi des canines saillantes, mais que, clairement, cela ne suffit pas à en faire un yaksha. On ne peut ignorer la puissance du symbolisme impérial dès lors que les douze yaksha apparaissent dans un contexte de formation d'un gouvernement de style chinois dans la province de Yamato. Ce n'est pas l'appartenance ethnique de ces douze figures qui importe en soi, mais leur signification en tant qu'esprits démoniaques convertis sur un plan bouddhique et politique. La comparaison que propose Asanuma avec des œuvres chinoises et coréennes, parmi lesquelles le temple souvent mentionné des quatre rois célestes, Sacheonwangsa, fondé en 679 à Gyeongju, capitale de Silla, est à mon sens peu concluante, car les temples coréens suivent eux-mêmes un style venu d'Asie du Sud ou d'Asie du Sud-Est ${ }^{65}$.

Si les douze yaksha sont effectivement représentés avec les caractéristiques ethniques des Chams, c'est que les Japonais en ont vu, qu'ils ont imité des représentations déjà existantes, ou qu'ils ont lu des témoignages. Le commerce maritime et les échanges 
diplomatiques rendent chacune de ces options possible. Les émissaires qui allaient du Japon vers la Chine des Tang et vers les trois royaumes de Corée ou inversement pouvaient emprunter différentes voies de mer au VII siècle, surtout dans les années 660, marquées par des conflits contre l'alliance des Tang et des Silla. Après la chute de Baekje en 660, la quatrième ambassade japonaise, envoyée à Chang'an en 659, a assisté au pardon et à la libération du roi Uija avant de revenir à Tsukushi (Kyūshū) en 661. Les forces japonaises ont ensuite apporté leur soutien à Baekje dans la bataille de Baekgang-gu (jp. Hakuson-ko) de 663, où elles ont été vaincues. Enfin, après la chute de Goguryeo (Koguryo) en 668, la septième ambassade de 669 a été présentée dans les sources chinoises comme celle qui avait salué la victoire de la Chine. Plus aucune délégation vers la Chine ni vers la Corée n'est partie entre 669 et 702. Dans l'autre sens, cinq missions chinoises ont embarqué vers le Japon depuis la commanderie d'Ungjin (une colonie fondée par les Tang pour le gouvernement de l'ancien royaume Baekje) entre 664 et $671^{66}$. Les migrations de réfugiés de Baekje ont eu un impact sensible sur la culture visuelle du Japon, en particulier dans l'iconographie des piédestaux. De grandes quantités de réfugiés, coréens et non-coréens, sont entrés dans l'archipel pendant ces années tumultueuses et ont été soumis à du travail forcé à une époque où le Japon se dotait d'un gouvernement impérial de style Tang. Une guerre de succession, la révolte Jinshin (Jinshin no ran 壬申年之亂), s'est ouverte en 672, suite à la mort de l'empereur connu sous son nom posthume de Tenchi Tennō 天智天皇 (626-672; r. 662-671), entre son fils, le prince Ōtomo 大友皇子 (648-672), et son frère cadet, le prince Ōama 大海人 皇子 (631 ?-686), qui allait devenir Tenmu Tennō. Les Tang, qui avaient leur ambassade près du port de Hakata (aujourd'hui Fukuoka), sur l'île de Kyūshū, n'en ont rien su, et Tenmu a ensuite été salué comme le sauveur du Japon.

Je propose de considérer les douze yaksha comme représentatifs de la politique impériale des commanditaires de Yakushi-ji vis-à-vis des frontières - une politique visant à soumettre et à assujettir au tribut, à conquérir et à dominer, dans le sens d'une souveraineté de style Tang -, mais aussi de la situation " domestique " de la province de Yamato vis-à-vis de ses frontières nord et sud grâce à l'image des " autres ", les forces du mal converties, à la fois yaksha et kunlun nu au service du Maître de Médecine des sūtra. Ces cosmologies de la loi juste et des êtres vertueux sous la loi bouddhique sont encore "surmontées " par ce qui apparaît comme un parallèle entre le souverain et Bouddha.

Le style des douze yaksha et de la créature hybride peut être utilement rapproché de certains éléments découverts sur la route de la soie en Asie centrale et en Asie du Sud au regard de la représentation des esprits démoniaques et des êtres convertis, même si aucune source particulière ne peut être attestée. Contrairement à Asanuma et à d'autres, qui ont cherché une origine ethnique pour chaque figure, je pense qu'elles représentent plutôt un amalgame et que, comme tous les autres éléments dans l'iconographie des piédestaux en mont Meru, leur signification ne peut pas être " réduite " à leur lien avec une source particulière, mais seulement évaluée dans le cadre d'un cosmoscape entendu comme combinaison d'éléments. Encore une fois, la représentation des douze yaksha et de la créature hybride vient surmonter celle des quatre animaux, gardiens de la loi juste, du bouddhisme vertueux, et de la stabilité du cosmos. Nagaoka et d'autres ont raison de souligner la présence des quatre animaux sur les tombes peintes de la région d'Asuka aux alentours de l'an $700^{67}$. S'il met l'accent sur leur association avec la mort et avec d' " autres mondes " (isekai 異世界) dans cette iconographie funéraire, je préfère pour ma part interpréter les quatre animaux dans deux tombes d'époque connues (les kofun de Takamatsuzuka et de Kitora, découvertes dans la préfecture de Nara, respectivement en 1972 et en 1983) comme un moyen de délimiter le cosmos et 
de symboliser ses gardiens, une interprétation attestée dans les textes classiques chinois et encore confirmée par des objets rituels de la cour.

Si des " étrangers " (des non-Han, à la peau noire) figurent aussi dans les peintures murales du kondō de Hōryū-ji (vers 700) et dans d'autres œuvres où ils font partie de la suite du Bouddha, leur représentation sur le piédestal, où ils apparaissent assis dans des niches closes et communiquant l'un avec l'autre, est d'un genre très différent. Les porteurs de pagne aux cheveux bouclés et aux corps amples et souples portent des symbolismes divers selon qu'on les envisage dans une perspective politique, bouddhique, ou, plus largement, cosmologique. Leur signification pré-bouddhique, associée au mont Meru et à Kubera, et leur lien probable avec les yaksha des sūtra - le mal converti en bien - sont ici réinterprétés dans une perspective bouddhique. Si la tradition artistique chinoise représentait une diversité de non-Han, les peuples bouddhistes du Centre, du Sud et du Sud-Est de l'Asie apparaissaient littéralement, dans la période d'élaboration de l'idéologie impériale sous la dynastie Tang, avec ses notions du centre et de la périphérie, du souverain et de ses sujets, du Han et de l' "autre ", comme des incarnations de l'être maléfique converti. Les douze yaksha du piédestal symbolisent ainsi les caractéristiques du centre et de la périphérie ethniques, du gouvernant et du gouverné, tout en fonctionnant en tant qu'entités "bouddhiques ". Comme tous les étrangers qui venaient à la cour de Yamato, les Chinois Tang, les Coréens des trois royaumes, et les autres continentaux charriaient toujours une menace potentielle.

Nagaoka Ryūsaku propose une interprétation du piédestal comme construction symbolique destinée à afficher les ambitions (negau 願う) de sinicisation des gouverneurs de Yamato. Il souligne pourtant que les empereurs japonais avaient une vision similaire de leurs propres "frontières »- le Nihon shoki semble ainsi évoquer la présence de sculptures stylisées du mont Meru (Shumisenzō 須弥山像) dans les jardins des palais de la région d'Asuka (avant que la capitale ne soit installée à Fujiwara) ${ }^{68}$ - et remarque que les tributs remis par des émissaires d'Ezo (Emishi 蝦夷) et notamment du sud d'Hayato (Kyūshū) dans des sites comme Yutsuki 斎㭇 (à l'ouest d'Asukadera), alignaient idéologiquement les gouverneurs de Yamato avec les gouverneurs chinois ${ }^{69}$. Pour lui, cette allégeance est politique : elle tient sans doute à la menace représentée par Ezo, et va dans le sens d'un apaisement vis-à-vis d'Asuka. Il est dès lors possible que les douze yaksha renvoient aux " peuples des frontières " de l'archipel, qui faisaient aussi figure d'étrangers, englobés dans le kunlun $n u$.

La présence des quatre animaux juste au-dessous des douze yaksha ou figures d' « étrangers " vient confirmer cette idéologie impériale sinitique. Il n'est pas surprenant que les premières apparitions des quatre animaux à la cour datent de cette période, avec, d'une part, des peintures sur les murs des kofun de Takamatsuzuka et de Kitora, que les spécialistes font souvent remonter à la fin du VII ${ }^{\mathrm{e}}$ ou au début du VIII ${ }^{\mathrm{e}}$ siècle, de l'autre, des bannières réalisées pour la cérémonie du Nouvel An de 701 (Taihō 1, premier mois de la cinquième année du règne de Monmu Tennō, petit-fils de Jitō, deux ans avant la mort de celle-ci) selon un passage du Shoku Nihongi. Un émissaire japonais, parti le même mois pour la Chine des Tang, désormais gouvernée par l'impératrice Wu Zetian (Wuzetian 武則天；Zetian Wuhou, jp. Sokuten Bukō ; 624-705；r. 690-705), avait dû revenir au port pour repartir en 702. Dans le jardin du palais de Fujiwara :

L'empereur continua jusqu'au Daigokuden [la salle des audiences] et fit entrer la cour. À cette cérémonie, la bannière du Corbeau à trois pattes [le Soleil] fut hissée au portail : à gauche, avec la bannière du soleil, les bannières du Dragon azur et de l'Oiseau vermillon; à droite, avec la bannière de la Lune, les bannières de la Tortue noire et du Tigre blanc ${ }^{70}$. 


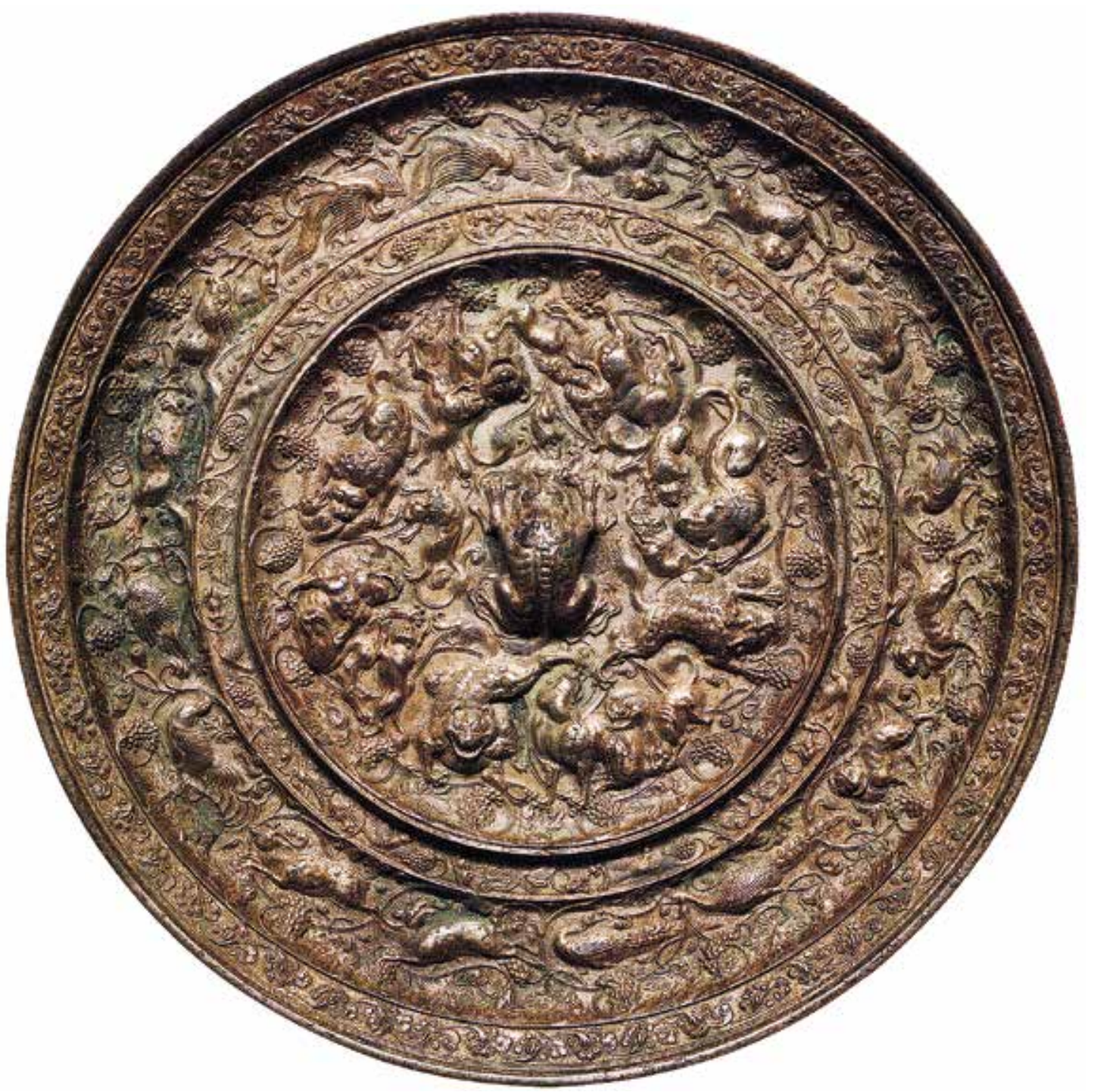

8. Kaijū budō kagami, miroir avec motifs de créatures marines et de grappes de raisin, dynastie Tang, Chine, ou période de Nara, Japon (VIIII siècle), cupronickel coulé, diamètre : $29,5 \mathrm{~cm}$, épaisseur à l'extrémité : $2 \mathrm{~cm}$, Chiba, Katatori-jingū (autel). Issu de Dai Kentōshi-ten..., 2010, cité n. 71, p. 182, fig. 181.
La cérémonie avait été prévue en l'honneur de dignitaires probablement venus du « royaume récemment unifié de Silla ", même si les relations avec lui s'étaient dégradées. Ils sont présentés comme des «barbares" (ebisu 夷) des « frontières " (ban' $i$ 蕃夷), assujettis aux Tang et leur devant tribut ${ }^{71}$. En 701, les noms, les couleurs et les directions associés aux quatre animaux étaient sans doute bien établis et constituaient déjà un élément essentiel de la cosmologie au Japon, comme le montrerait plus tard leur rôle dans l'orientation des villes et des appareils rituels. Ce passage du Shoku Nihongi marque par ailleurs la première occurrence du mot tennō apposé au nom du gouverneur ; la première occurrence du mot tennō apparaît quant à elle sur une tablette de bois (mokkan 木簡) que l'on fait remonter au règne de Tenmu. Si le piédestal du Bouddha de Yakushiji reste aujourd'hui incompris, c'est, je crois, principalement parce que les spécialistes 


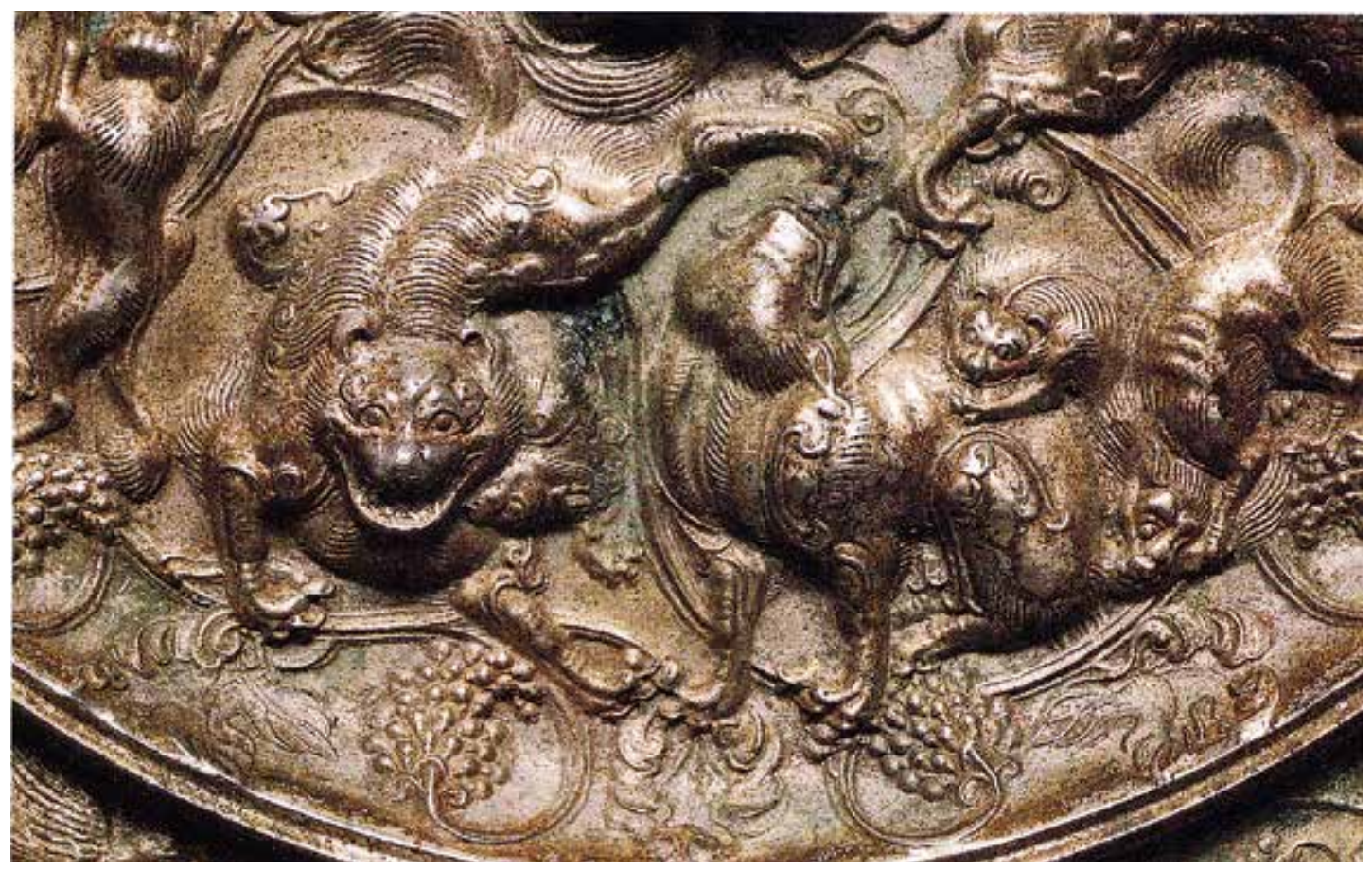

se sont concentrés sur la date de sa réalisation et sur les sources de son iconographie, sans le rapporter à la vision du monde du VII ${ }^{\mathrm{e}}$ siècle ni à ses précédents en termes de piédestaux en mont Meru, et sans se demander pourquoi des motifs " non-bouddhiques " avaient pu paraître appropriés sur l'icône d'un temple de l'importance de Yakushi-ji.

Le dernier motif qu'il nous reste à étudier est celui des entrelacs de vignes sur le plateau supérieur, où repose le Bouddha. Il s'inscrit dans une tradition iconographique attestée en Chine sous le règne de l'impératrice Wu Zetian, où les grappes de raisin apparaissent comme des fruits propitiatoires et féconds, nouveaux et encore exotiques. Les entrelacs de vignes ne sont pas plus caractéristiques de l'art bouddhique japonais que les quatre animaux $^{72}$. Il me semble qu'on peut envisager qu'ils soient issus des miroirs ornés de motifs de grappes de raisin et de créatures marines, courants en Chine au milieu et à la fin de la dynastie Tang et dans le Japon du VIII ${ }^{\mathrm{e}}$ siècle, ainsi qu'en témoigne par exemple le Kaijū budō kagami 海獣葡萄鏡 ${ }^{73}$ (fig. 8). Rappelons que les miroirs Tang ont été introduits au Japon pendant la période Kofun et qu'on en a découverts, suspendus au plafond et la face miroitante orientée vers l'extérieur, dans les tumuli funéraires (kofun) de la période Asuka et dans les pavillons de temples du VIII ${ }^{\mathrm{e}}$ siècle (ainsi de Kanzeon-ji, à Fukuoka, sur l'île de Kyūshū, ou du Hokkedō de Tōdai-ji, à Nara). En Chine, ils étaient plus courants dans les tombes, où d'autres objets réfléchissants pouvaient être utilisés $^{74}$. Les miroirs chinois étaient aussi produits pour un usage personnel, et, à la question de savoir si le mélange de motifs et de symboles cosmologiques propitiatoires qui en ornait le dos avait une fonction principalement ou exclusivement décorative, je penche davantage pour la première hypothèse. Le piédestal en mont Meru est en soi un cosmoscape. Imaginons que nous l'aplatissions au sol : le monde liminaire, avec les douze yaksha et les quatre animaux, figurerait ainsi au centre, tandis que les entrelacs de vignes représenteraient le bord extérieur, exactement comme au dos des miroirs. Dans le Kaijū budō kagami, de fabrication chinoise ou japonaise, la section médiane 
est occupée par la créature marine ainsi que par des lions et des lionceaux qui évoluent dans des motifs de vigne et de grappes de raisin. Elle est entourée de trois anneaux, dont le premier est occupé exclusivement par des grappes de raisin, le deuxième, par un motif de vigne et de grappes de raisins où évoluent divers oiseaux (canard, phénix, paon, et oiseaux paradisiaques), et le troisième, à l'extérieur, par un motif délicat de nuages qui filent. Avant la popularisation des miroirs à quatre ou cinq créatures marines, la Chine en produisait avec des représentations des quatre animaux et des dix tiges célestes, souvent dans des symboles horoscopiques. Les motifs des bords extérieurs enveloppent le cosmoscape à l'intérieur et, que le tout soit doué de trois dimensions ou aplati de cette manière hypothétique, on voit qu'il fonctionne de la même façon.

En conclusion, cet article a formulé plusieurs hypothèses originales. Premièrement, il a présenté le piédestal du Bouddha de Yakushi-ji comme le support d'un système de symboles complémentaires, d'un double sens associant le pouvoir guérisseur du Bouddha de la Médecine et le pouvoir de conversion et de protection du bouddhisme vis-à-vis des forces du mal aux frontières de l'empire, en parallèle avec les nombreux éléments symboliques et magiques de son programme iconographique situés dans la perspective d'une cosmologie qui définit les notions de centre et de périphérie, la loi juste, et l'éradication du mal. Deuxièmement, il a renouvelé l'interprétation des motifs non-bouddhiques et, plus généralement, des piédestaux en mont Meru, remettant ainsi en question les conceptions établies sur les rapports des hommes et, plus particulièrement, des bouddhistes avec le cosmos à la fin du VII siècle. Enfin, il a proposé une interprétation cohérente de l'iconographie du piédestal, en lien avec les ambitions de l'empire ainsi qu'avec les sources dont il disposait et avec les œuvres qu'il commandait à son propre usage. Cet article est ainsi le premier à étudier le rôle de l'art bouddhique japonais dans la perspective de l'élaboration d'un gouvernement impérial de style chinois de la même façon qu'on peut étudier les textes essentiels de cette époque pour se renseigner sur l'essor de l'autorité culturelle dans ce tournant qu'a été la période entre la fin du VII et le début du VIII ${ }^{\mathrm{e}}$ siècle. Le honzon de Yakushi-ji, à cheval entre deux capitales, entre deux temples et entre deux siècles, les incarne aussi bien les uns que les autres. À mi-chemin entre la Triade de Shaka de Hōryū-ji et le Grand Bouddha de Tōdai-ji, il s'agit d'une œuvre clé dont le cosmoscape, ou " cosmologie sous le Bouddha " a bien davantage à nous enseigner sur les pratiques religieuses de cette période et sur le mode de fonctionnement des icônes que ce qu'on a bien voulu voir jusqu'ici.

Cette contribution a été traduite de l'anglais par Étienne Gomez. 


\section{Cynthea J. Bogel}

Cynthea J. Bogel est professeure d'histoire de l'art japonais et des cultures visuelles bouddhiques d'Asie de l'Est à la Kyūshū University depuis 2012. Elle avait auparavant travaillé à I'University of Washington (Seattle), à l'University of Oregon, et au RISD Museum (Rhode Island). Ses domaines d'expertise sont la culture visuelle bouddhique, les estampes ukiyo-e ; les laques, les céramiques, les textiles, et la mode au Japon, et le patrimoine culturel. Lauréate, entre autres, de bourses de recherche du National Endowment for Humanities et de la Getty Foundation (Los Angeles), elle est fondatrice et rédactrice en chef du Journal of Asian Humanities at Kyūshū University (JAH-Q, à comité de lecture).

\section{NOTES}

Cet article a bénéficié du soutien de deux bourses de recherche Ailsa Mellon Bruce qui m'ont permis d'être reçue en résidence au Center for Advanced Study in the Visual Arts (CASVA, National Gallery of Art, Washington, D.C.) deux étés de suite, en 2016 et en 2017, ainsi que d'une subvention à la recherche scientifique (type B) de la part de la Japan Society for the Promotion of Science (JSPS) pour la période 2018-2023 ("The Representation of Wrath and Evil: Conceptions of the World and Expressions of the Wicked and the Converted in Ancient Japan ", réf. 18H00630). Je remercie nombre de mes collègues pour leurs commentaires éclairants sur les hypothèses que j'ai présentées lors de communications ou sous forme écrite. Je dois une mention particulière à Lindsey DeWitt, Torquil Duthie, Michael Jamentz, Bryan Lowe et Yoko Shirai pour leurs remarques sur les étapes préparatoires de cet article, ainsi qu'à Nagaoka Ryūsaku pour les discussions que nous avons eues ensemble et pour sa stimulante érudition.

1. La recherche assimile traditionnellement l'ancienne Nara à la capitale Heijōou Heijōkyō 平城京, mais certains chercheurs pensent que les deux premiers caractères, 平城, de même que 寧楽 et d'autres utilisés pour la capitale étaient tous prononcés « Nara ». Ici, le terme Nara est utilisé par commodité pour désigner à la fois la ville moderne et l'ancienne capitale Heijō 平城.

2. Nikkō bosatsu 日光菩薩, 317,3 cm, et Gakkō bosatsu 月光菩薩, $315,3 \mathrm{~cm}$.

3. Les trois mandorles dorées datent de 1635. Le Bouddha et son piédestal sont originaux mais peuvent remonter à des dates différentes, tout comme les bodhisattva.

4. Le mot jōroku 丈六 est composé de jō 丈 (un jō étant l'équivalent de dix shaku 尺 et un shaku représentant la taille d'un petit pied) et de roku 六 (《six »). Il évoque ainsi un total de seize shaku (approx. $485 \mathrm{~cm}$ ) qui correspond à la taille du Bouddha Śākyamuni selon d'anciennes références (deux fois la taille d'un très grand homme). La hauteur d'un Bouddha assis jōroku est d'environ
8 shaku, soit la moitié d'un Bouddha debout. Le premier Bouddha jōroku attesté au Japon est le honzon d'Asuka-dera 飛鳥寺 (alt. Hōkō-ji 法興寺, Asuka, Préfecture de Nara), daté de 609.

5. La triade a été déclarée Trésor national le 9 juin 1951 sous le nom de "Bronze doré de Yakushi Nyorai et deux bodhisattva, trois statues 》(銅造薬師如来及両脇 侍像 3躯). Sur son inscription dans la liste du patrimoine mondial de l'UNESCO, voir https://whc.unesco.org/fr/ list/870/ (en français).

6. Il existe une traduction anglaise d'un article écrit en japonais par Kuno Takeshi et Inoue Tadashi : "Study of the Yakushi-ji Triad in the Kondō, Yakushi-ji ", dans Acta Asiatica, vol. 1, 1960, p. 89-108. Parmi les publications en anglais, on trouve un chapitre approfondi sur Moto Yakushi-ji (p. 201-236) par Donald McCallum dans The Four Great Temples: Buddhist Archaeology, Architecture, and Icons of Seventh-Century Japan, Honolulu, University of Hawaï Press, 2008. Sur les icônes, voir Jiro Sugiyama, Classic Buddhist Sculpture: The Tempyō Period, Tōkyō, Kodansha International, 1982 ; Yui Suzuki, Medicine Master Buddha: The Iconic Worship of Yakushi in Heian Japan, Leyde, Brill, 2012 ; et Yui Suzuki, "The Aura of Seven: Reconsidering the Shichibutsu Yakushi Iconography », dans Archives of Asian Art, vol. 60, nº 1, 2010, p. 19-42. Les publications en japonais, comme je l'ai déjà signalé, sont nombreuses. Par commodité, je les citerai le cas échéant en note de bas de page.

7. Les historiographes n'écrivaient pas alors Fujiwarakyō mais « la capitale nouvellement construite " (shin'yakukyō ou aramashi no miyako 新益京), comme en témoigne par exemple l'entrée Jitō 5 (691).10.27 (ils appelaient par ailleurs le palais Fujiwarakyū ou Fujiwara no miya 藤原宮). Selon l'usage des spécialistes d'aujourd'hui, j'emploierai l'appellation de Fujiwarakyō.

8. Le site de Moto Yakushi-ji, partiellement mis à jour, peut être visité même si l'emplacement du pavillon principal est aujourd'hui occupé par un bâtiment. II se situe dans le quartier de Kidono-cho, à Kashihara, entre le mont Unebi et le mont Kaguyama.

9. Nagaoka fait ici exception. Nagaoka Ryūsaku 長岡 龍作, "Dainihen shumiza kō: Yakushi-ji kondō Yakushinyoraizō no daiza o megutte ", 第二編 須弥座考: 薬師寺金堂薬師如来像の台座をめぐって, Butsuzō shōgon ni arawareru haka mochīfu ni kansuru chōsa kenkyū 仏像荘厳にあらわれる墓モチ一フに関する 調査研究, Kenkyū seika hōkoku 研究成果報告, 2005, p. 23-36 (subvention à la recherche scientifique, type C [2] pour la période 2004-2006, réf. 14510073). Voir aussi Nagaoka, Hōryū-ji to Nara no jiin 法隆寺と奈良 の寺院, Tōkyō, Shōgakukan, 2012.

10. Voir Itō Shirō 伊東史朗, "Yakushi-ji kondō yakushisanzonzō 薬師寺金堂薬師三尊像 》, dans Kenkyū happyō to zadankai: Heijō-kyō ni okeru shūkyō bijutsu no sho mondai 研究発表と座談会: 平城京における 宗教美術の諸問題, Ueyama Shunpei 上山春平 (éd.), Bukkyō bijutsu kenkyū ueno kinen zaidan josei kenkyūkai hōkokusho 仏教美術研究上野記念財団 助成研究会報告書, vol. 19, 1989, p. 12, p. 10-14.

11. Arjun Appadurai, "Disjuncture and Difference in the Global Cultural Economy », dans Theory, Culture 
\& Society, vol. 7, 1990, p. 295-310 (en ligne, DOI : 10.1177/026327690007002017).

12. Voir, par exemple, Asanuma Takeshi 浅湫毅, "Yakushi-ji kondō honzon daiza no igyōzō nit suite " 薬師寺金堂本尊台座の異形像について, dans Ars buddhica, Bukkyō geijutsu 仏教芸術, vol. 208, 1993, ill. 5 et 14, p. 53-71, et Nagaoka, 2005, cité n. 9, p. 23-36.

13. Hayashi Ryoichi 林良一, «Yakushi-ji honzon daiza no budō karakusa mon » 薬師寺本尊台座の葡萄唐草 文, dans Kokka 國華, no 810, 1959, p. 342-351, et Tōyō bijutsu no sōshoku mon'yō: Shokubutsumon hen 東洋 美術の装飾文様: 植物文篇, Kyōto, Dōhōsha, 1992.

14. Nagaoka, 2005, cité n. 9, p. 23-26.

15. Herman Ooms, Imperial Politics and Symbolics in Ancient lapan: The Tenmu Dynasty, 650-800, Honolulu, University of Hawaï Press, 2016, p. XIX. Selon Ooms, le taoïsme a joué un rôle important dans la "symbolique du rituel et du cérémonial politique » sous le règne de Tenmu, "même si ses attestations dans les annales restent vagues dans le meilleur des cas » (p. XVIII). Comme de nombreux spécialistes, je pense que ce que Ooms appelle «taoïsme » dans le Japon ancien correspond davantage à l'idée de " religion populaire chinoise ».

16. Cynthea J. Bogel, "Cosmoscapes: Interpreting Buddhist Imagery in Ancient Japan ", dans CENTER 38, Record of Activities and Research Reports, June 2017May 2018, Washington, D.C., CASVA, 2018, p. 59-63 ; et "Sovereign and Cosmology in Two Capitals: An EighthCentury Buddhist Icon at Yakushi-ji », dans CENTER 37, Record of Activities and Research Reports, June 2016May 2017, Washington, D.C., CASVA, 2017, p. 56-59. J'ai aussi prononcé plusieurs communications sur le piédestal, parmi lesquelles : "Cosmoscapes and Hybrid Traces on an Eighth-Century Japanese Buddhist Icon ", EuroAsian Connections, Congrès annuel du Center for Global Asia, NYU Shanghai, août 2018 ; " Imagery ca. 700 Japan: Cosmoscapes of Rule and Religion", Annual Shinto Lecture, UCLA (Center for Buddhist Studies, Center for the Study of Religion), mai 2018 ; "Cosmology Beneath the Master of Medicine: The Eighth-Century Pedestal at Yakushi-ji, Nara », Japan Colloquium Series, Yale (Council on East Asian Studies at Yale University), novembre 2016.

17. Selon Michael Como, "parmi les facteurs les plus importants stimulant la production littéraire à la cour, on note une tentative de reconfigurer à la fois I'histoire collective et les pratiques rituelles en tant qu'elles étaient liées aux lignages et aux centres cultuels dans l'archipel japonais [...]. Je formule donc l'hypothèse que la cour était tout aussi bien consommatrice que productrice de nouvelles formes idéologiques et rituelles. » (Weaving and Binding: Immigrant Gods and Female Immortals in Ancient Japan, Honolulu, University of Hawaï Press, 2009, p. XVII).

18. Watsuji Tetsurō 和辻哲郎, Pilgrimages to the Ancient Temples in Nara (1919), Honolulu, University of Hawaï Press, 2012 (en japonais : Koji junrei 古寺巡礼, Tōkyō, Iwanami Shoten, 2009).

19. Pour des hypothèses mesurées sur Tōdai-ji et sur sa culture matérielle, voir par exemple Michael Cunningham et al., Buddhist Treasures from Nara, Manchester, VT, Hudson Hills, 1998 ; et Sugiyama
Jirō, Classic Buddhist Sculpture, Samuel Morse (intro. et trad. angl.). Bryan D. Lowe, Ritualized Writing: Buddhist Practice and Scriptural Cultures in Ancient Japan, Honolulu, University of Hawaï Press, 2017, remet brillamment en question I'importance excessive traditionnellement accordée à l'État et à l'action de I'État dans les pratiques religieuses pendant la période Nara. Como admet l'importance relative « des lignées d'immigrants et de leurs habitudes et préoccupations sur le plan technique et rituel "à la cour de Yamato, dans Michael Como, 2009, cité n. 18. Pour les études en japonais sur Tōdai-ji, voir Mizuno Keizaburō 水野 敬三郎, Okada Hideo 岡田英男, et Asai Kazuharu 浅井 和春 (dir.), Tōdai-ji to Heijōkyō: Nara no kenchiku, chōkoku 東大寺と平城京: 奈良の建築・彫刻, Tōkyō, Kōdansha, 1990 ; sur les icônes du début du VIII' siècle et pour des perspectives différentes en histoire de l'art, voir Tsuji Nobuo 辻惟雄, Nagaoka Ryūsaku 長岡 龍作, Izumi Takeo 泉武夫, Yamashita Yūji 山下裕二 et Itakura Masaaki 板倉聖哲 (dir.), Hōryū-ji to Nara no jiin: Asuka, Nara jidai 1 法隆寺と奈良の寺院: 飛 鳥·奈良時代1, Nihon bijutsu zenshū 日本美術全集, vol. 2, Tōkyō, Shōgakukan, 2012. Les dix-sept volumes publiés à ce jour des Za gureito budda shinpojiumu ronshū ザ・グレイトブッダ・シンポジウム論集 (Papers From the Great Buddha Symposium : GBS Jikkō linkai, 2003-) sont aussi particulièrement utiles.

20. Torquil Duthie, Man'yōshū and the Imperial Imagination in Early Japan, Leyde, Brill, 2014, p. 3.

21. Le terme de "souverain(e) céleste " (" heavenly sovereign ») a été introduit par l'historienne Joan Piggott comme alternative à « empereur » ou " impératrice ». Pour une excellente synthèse sur cette étymologie, sur la première occurrence historique du terme, et sur sa signification, voir Duthie, 2014, cité n. 20, p. 87-94. Sur le système impérial, voir Tōno Haruyuki 東野治之, 《Tennōgō no seiritsu nendai ni tsuite 》 「皇号の成立 年代について」, dans Shoku Nihongi kenkyū 『続日 本紀研究』, vol. 144-145, mai 1969 (réimprimé dans Shōsōin monjo to mokkan no kenkyū 『正倉院文書 と木簡の研究』, Tōkyō, Hanawa shobō, 1977).

22. Le mont Meru, connu aussi sous le nom de mont Sumeru, est reconnu dans les traditions hindoue, jaïne, et bouddhiste à travers toute l'Asie.

23. Voir l'entrée shumidan dans Bukkyō daijiten 佛 教大辞典, Mochizuki Shinkō 望月信亨 (éd.), Tōkyō, Bukkyō Daijiten Hakkōjo, 1933, vol. 3, p. 2515. Sur les deux piédestaux de Hōryū-ji discutés ici, voir l'article de Nagaoka Ryūsaku 長岡龍作, "Hōryūji: bijutsu to inori » 法隆寺一美術と祈り, dans Tsuji Nobuo et al. (dir.), 2012, cité n. 19, p. 170-188. Ce même volume contient des photographies des trois piédestaux discutés ici (Bouddha de Yakushi-ji, Triade de Shaka et Tamamushi no zushi) ainsi que des photographies infrarouges de deux d'entre eux (Bouddha de Yakushi-ji, Bouddha de la Triade de Shakai) : voir les illustrations $\mathrm{n}^{\circ} 8,10,11,14$ et 88 (n.p.), ainsi que les illustrations en noir et blanc, p. 175-177. En anglais, voir Akiko Walley, "Flowers of Compassion: The Tamamushi Shrine and the Nature of Devotion in Seventh-century Japan », dans Artibus Asiae, vol. 72, n 2, 2012, p. 265-322.

24. La qualification de « miniature de palais » est attri-

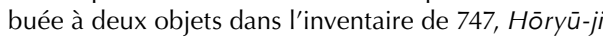


garan engi narabini ruki shizaichō 法隆寺伽藍緑起并 流記資財帳.

25. Tout en rappelant que cette référence a été remarquée par Ono Genmyo 小野玄妙 en 1927 (voir le chapitre « Kodai Chūō Ajia no Bukkyo geijutsu ni taisuru gigi »古代中央亜細亜の仏教藝術に対する疑議, dans Daijö Bukkyo geijutsushi no kenkyū 大乗仏教芸術史 の研究』, Tōkyō, Yūzankaku, 1927, p. 90-117), Walley note que le palais du Dragon, même s'il est inspiré d'un sūtra (en l'occurrence le Sūtra Bussetsu Kairyūōkyō 仏説 海龍王経, dans Takakusu Junjirō, Watanabe Kaigyoku, et. al. (dir.),Taishō shinshū daizōkyō, Tōkyō, Taishō Issaikyō Kankōkai,1924-1932, vol. 215, n 598), est situé sur le panneau dans la mer du mont Meru (voir Walley, 2012, cité n. 23, p. 265-322, en particulier p. 308-313). Elle souligne par ailleurs les rapports entre les peintures des panneaux du Tamamushi no zushi et le thème de la charité (fuse 布施 ou danharamitsu 檀 波羅蜜), la pratique de l'aumône étant fondamentale dans le bouddhisme, et voit dans le Sūtra du lotus de la grande compassion (Hikekyō; sk. Karuna-pundarlka sūtra) le cadre assurant le lien thématique entre ces diverses peintures.

26. Voir Nagaoka, 2005, cité n. 9, qui, comme noté plus haut, voit l'ensemble formé par le Bouddha de Yakushiji et son piédestal comme une déclaration visuelle de sinification et d'allégeance à la Chine (voir p. 23 pour le piédestal et p. 28-29 pour le commentaire).

27. Voir Nagaoka, 2012, cité n. 23.

28. Des gravures de pétales, parmi lesquelles une représentation du mont Meru, peuvent être consultées en ligne à l'adresse suivante : http://niihamajin19.web.fc2. com/syumisenn.html.

29. Masuo Shin'ichirō 増尾 伸一郎, “Chinese Religion and the Formation of Onmyōdō ", dans Japanese Journal of Religious Studies, vol. 40, no 1, 2013, p. 19-43, p. 20.

30. Livia Kohn, "Buddhist-Daoist Interactions in Medieval China ", dans Mario Poceski (dir.), The Wiley Blackwell Companion to East and Inner Asian Buddhism, Hoboken, NJ, John Wiley \& Sons, Ltd., 2014, p. 340-359.

31. Nihon shoki, Tenmu 9 (680).11.12 : 癸末。皇后。體 不豫。則爲皇后誓願之。初興薬師寺。仍度一百僧。由是 得安平癸末. La future Jito Tennō était alors princesse consort, mais le texte, ayant été rédigé après sa mort, la qualifie déjà d'impératrice. "Renoncer au monde » signifie prononcer des vœux monastiques.

32. Une « [Grande] Assemblée Sans Délai » (Musha daie 無遮大会, ou Daimushae 大無遮大会) est rapportée dans Jitō 2 (688).1.8, 「設無遮大会於薬師寺」. Un peu plus d'un an auparavant, en 686.12.19, le Nihon shoki relate un rite similaire pour le repos de Tenmu cent jours après sa mort, célébré à la fois à Daikandai-ji, Asukadera, Kawara-dera 川原寺, Owarida Toyura-dera 小墾 田豐浦寺, et Sakada-dera 坂田寺. Yakushi-ji n'étant pas mentionné, certains spécialistes en concluent que le complexe n'était pas adapté pour cette pratique rituelle, autrement dit qu'il n'était équipé ni d'un kondō ni d'un honzon.

33. Mizuno Keizaburō 水野敬三郎, conversation personnelle sur la nécessité des bâtiments essentiels pour le rituel de 688, novembre 2014. Voir Ōhashi
Katsuaki 大橋一章, Yakushi-ji 薬師寺, Ōsaka, Hoikusha, 1986 ; Naitō Sakae 内藤栄, «Sōron: Hakuhō no bijutsu » 総論: 白鳳の美術, dans Hakuhō: Hanahiraku Bukkyō bijutsu: Kaikan hyakunijūnenkinen tokubetsuten 白鳳: 花ひらく仏教美術 : 開館120年記念特別展 [Hakuhō, The First Full Flowering of Buddhist Art in Japan], cat. exp. (grande exposition célébrant le $120^{\mathrm{e}}$ anniversaire du Musée national de Nara, 2015), Nara, Musée national de Nara, 2015, p. 6-16, p. 12. Voir aussi Ōhashi Katsuaki 大橋一章, «Fujiwarakyō Yakushi-ji zōei kō » 藤原京 薬師寺造営考, dans Ronsō Bukkyō bijutsushi 論叢仏 教美術史, Tōkyō, Yoshikawa Kōbunkan, 1986, p. 321352. Un honzon brodé du Bouddha Amida a été réalisé pour l'un des bâtiments, sans doute le pavillon des prières, en 692.

34. Voir, par exemple, Mori Ikuo 森郁夫, «Kodai jiin no iken to saiken mondaiten » 古代寺院の移建と再建の 問題点, Kyōto kokuritusu hakubutsukan [Musée national de Kyōto], Gakusō 学叢, vol. 25, 2003, p. 123-132.

35. Les témoignages : Nara Kokuritsu Bunkazai Kenkyūjo 奈良国立文化財研究所, Yakushi-ji hakkutsu chōsa hōkoku 薬師寺発掘調査報告, Nara, Nara Kokuritsu Bunkazai Kenkyūjo, 1987. Voir Hanatani Hiroshi, « MotoYakushiji no hakkutsu chōsa » 本薬師寺の発掘調査, dans Ars Buddhica, vol. 235, 1997, p. 57-80.

36. Le colloque du 30 novembre 2019 était intitulé "Heijō Yakushi-ji o meguru shinpojiumu 'garan o utsusu' koto no imi o kangaeru » 平城薬師寺をめぐる シンポジウム『伽藍 (がらん) を移す』ことの意味を考 える」. À côté des deux communications discutées dans l'article, l'historien de l'architecture Unno Satoshi 海野聡 a évoqué le temps nécessaire à la construction du seul bâtiment de Yakushi-ji existant au VIII e siècle, la pagode de l'Est (vers 730), dans "Succession et innovation en matière de style architectural dans la pagode de l'Est de Yakushi-ji (Yakushi-ji tōtō ni miru kenchikuyōshiki no keishō to kakushin「薬師寺東塔にみる建築様式 の継承と革新」) 》, et l'historien Yoshikawa Shinji 吉川 真司 a présenté une communication intitulée "Le temple Garan et son territoire dans les régions d'Asuka et de Fujiwara après la dissolution de la capitale (Haitogo no Asuka Fujiwara chiiki no garan to jichi「廃都後の 飛鳥・藤原地域の伽藍と寺地」)». Yoshikawa a fait état de preuves substantielles en faveur de l'hypothèse selon laquelle Moto Yakushi-ji a conservé sa fonction religieuse en tant que temple d'origine, comme ce fut le cas de Moto Gangō-ji 本元興寺 (Asuka-dera). Les deux modérateurs de la dernière partie du colloque étaient Hida Romi 肥田路美 (Waseda University, Tōkyō) et Takahashi Teruhiko 高橋照彦 (Osaka University). Voir : http://www.butsugei.org/synpo20191130youkou.pdf.

37. Dans sa communication, Ishida a également présenté une synthèse des opinions de Hanatani Hiroshi et d'autres qui avaient déjà soutenu, sur la base d'analyses de tuiles découvertes dans la pagode de l'Ouest, que Moto Yakushi-ji était resté actif au moins jusqu'à la fin de la période Nara. Hanatani Hiroshi 花谷浩, «Tera no kawara zukuri to miya no kawara zukuri » 寺の瓦 作りと宮の瓦作り, dans Kōkogaku kenkyū 考古学研究， vol. 158, 1993, p. 72-93 ; et Hanatani, 1997, cité n. 35.

38. La mère de Monmu, Genmei Tennō, a elle aussi exercé son règne depuis Fujiwarakyō (697.8.1). 
39. Le Daikandai-ji 大官大寺, que l'on peut sans doute considérer comme le temple impérial le plus important d'Asuka, a été construit en 677 dans la capitale de Fujiwara (il a eu d'autres emplacements et d'autres noms ; à l'occasion de son transfert à Nara, il a été rebaptisé « Daian-ji » en 738) puis transféré dans la partie Est de la capitale, tandis que le Yakushi-ji était dans la partie Ouest (selon un axe central nord-sud). Selon les Daian-ji engi 大安寺緑起 de 747 : 《Le temple [Daikandai-ji] et [sa] statue de Bouddha jōroku ont été transférés à Nara [和 銅三年庚戌移伽藍及丈六仏像等於平城] ». Le caractère utilisé pour " transférés » est 移. Pour le document, voir Hanawa Hokinoichi 塙保已一(dir.), Gunsho ruijū dai nijū yon shū, shakke bu 群書類聚第24輯、 釈家部, Tōkyō, Zokugunshoruijū Kinseikai, 1980, vol. 435, p. 394. Pour une discussion sur le transfert du honzon du Daikandai-ji, voir deux articles de Tanaka Megumi 田中惠 : «Daian-ji shakazō no shūhen (sono ichi): Nihon bukkyō chōkoku ni okeru 'shūkyō zōkei' to 'shinkō zōkei' ni tsuite " 大安寺釈迦像の周辺: 日本 仏教彫刻における「宗教造形」と「信仰造形」につい て, dans Iwate Daigaku Kyōikugakubu kenkyū nenpō, vol. 55, nº 1, 1995, p. 59-74; il s'agit là de la première partie (sono ichi) de l'étude, dont la deuxième partie (sono ni) a été publiée dans le même journal, vol. 55, $\mathrm{n}^{\circ} 2,1996$, p. 29-43. Parmi les autres objets matériellement « transférés " à Nara, on trouve des tuiles d'Asukadera, qui ont transité vers Gangō-ji 元興寺.

40. 已上持統天皇奉造請坐者. Le document discuté par Naitō est un témoignage (ruki 流記) contenu dans le Yakushi-ji engi, "Kondōjō » 金堂条. Voir Naitō Sakae 内藤栄, "Yakushi-ji engi kondō jō ni okeru ryūki inyō ni tsuite» 薬師寺縁起金堂条における流記引用 について, dans Rokuon zasshū: Bulletin of the Nara National Museum 鹿園雜集: 奈良国立博物館研究紀 要, vol. 15-16, 2015, p. 15-27, p. 17. Naitō trouve des formulations similaires dans le Hōryū-ji garan engi narabini ruki shizaichō 法隆寺伽藍緑起并流記資財帳 (où l'on trouve 敬造請坐者), tandis que le Daian-ji Ruki 大安寺流記 utilise 奉造而請坐者. Comme les témoignages de la période Heian ne recourent pas à ce genre de termes, Naitō conclut que le terme utilisé dans le ruki du Yakushi-ji engi date de la période de Tenpyō, et que le choix du titre posthume de Jitō est la seule altération apportée, pour la commodité du lecteur de l'époque de Heian. La validité du ruki comme document Tenpyō authentique est niée à la fois par Sekino (1901), Adachi (1939-1945), Machida Kōichi 町田甲一 (1975), et Ōhashi Katsuaki 大橋一章 (voir Yakushi-ji 薬師寺, Ōsaka, Hoikusha, 1986). Naitō s'est appuyé sur les travaux de l'historien Tōno Haruyuki 東野治之, professeur émérite à l'université de Nara (voir, par exemple, «Bunkenshiryō kara mita yakushi 文献資料からみ た薬師寺 », dans Yamato koji no kenkyū 大和古寺の研 究, Tōkyō, Hanawa Shobō, 2011, p. 179-200).

41. La communication de Fujioka était intitulée : « Les statues de Yakushi-ji et de Yamada-dera en termes de technique, d'alliage, et de style (Gihō kinzoku sosei, yōshiki kara mita Yakushi-ji-zō to Yamadadera-zō 技法·金属組成 ・様式からみた薬師寺像と山田寺像)》. Les références de sa bourse de recherche actuelle (JSPS, Kiban A, 20182022) et des autres bourses dont il a bénéficié pour l'étude de la statuaire antique peuvent être consultées à l'adresse suivante : https://nrid.nii.ac.jp/ja/nrid/1000070314341/.
42. Les œuvres antérieures à la période Nara sont : $1^{\circ}$ la tête de l'icône principale jōroku (disparue) de Yamadadera 山田寺, aujourd'hui à Kōfuku-ji 興福寺 (une rare œuvre datée de la période Asuka de 685.3.25 [Tenmu 14]) $; 2^{\circ}$ les deux bodhisattva (env. 700) du kondō de l'Est de Kōfuku-ji 興福寺東金堂 $(94.6 \%$ de cuivre) ainsi que la statue de Shō Kannon 聖観音 du Tōindō de Kōfuku-ji 東院堂 (96\% de cuivre). Toutes contiennent davantage d'impuretés (fer, plomb, bismuth) que le Bouddha de Yakushi-ji. Techniques utilisées : analyse par fluorescence à rayons $\mathrm{X}$ et mesure tri-dimensionnelle (Keikō X-sen bunseki to sanjigen keisoku 蛍光X線分析と三次元計測).

43. Les réparations exécutées sur le piédestal au début des années 1950 ont donné lieu à une publication donnant le détail du démantèlement en 1958, suivie de plusieurs autres articles et enfin d'une monographie de Machida sur Yakushi-ji en 1984 : Yakushi-ji shūri i'inkai (ed.), 薬師寺修理委員会 編, Yakushi-ji Kokuhō Yakushi sanzon tō shūri kōji hōkoku sho 薬師寺国宝 薬師三尊等修理工事報告書, Nara, 薬師寺修理委員会, 1958 (en ligne, DOI : 10.11501/8798699); Machida Kōichi 町田甲一, Yakushi-ji 薬師寺, Tōkyō, Gurafusha, 1984, p. 115-123 (la reconstitution de Machida est à la p. 117 ; l'autre est issue de Tamura Yoshinaga 田村吉 永, «Yakushi-jidōtō honzon zōryū shinkō » 薬師寺堂 塔本尊造立新考, dans Bukkyō geijutsu「佛教藝術」， $\mathrm{n}^{\circ} 15,1952$, p. 65-83). La partie ouest (celle de gauche pour le spectateur) est plus élevée que celle de l'est (à droite), avec une différence d'environ 1,32 cm (mesure basée sur Machida, 1984, p. 116, fig. 65).

44. Une partie du piédestal était en train de s'effondrer. Voir Machida, 1984, cité n. 43, commentaire p. 115 et suiv., fig. 23 (vue frontale sans la section supérieure) et fig. 24 (vue du bord coupé depuis le dessus).

45. 始建薬師寺東塔 dans le Fusō ryakki 扶桑略記. Voir Kuroita Katsumi 黑板勝美, Fusō ryakki, Tōkyō, Yoshikawa Kōbunkan, 1999, et Kuroita, Fusō ryakki. Teiō hennenki 扶桑略記: 帝王編年記 (Kōen-hen 皇圓 編 et Eiyū-hen 永祐編), Yoshikawa Kōbunkan, 2007. Voir également Chikamichi Ōe 大江親通, 1140 (Hōen 保延 6), "Private Journal of a Pilgrimage to the Seven Great Temples», Nana-daiji junrei shiki 七大寺巡私 記, dans Kokan bijutsu shiryo: Jiin hen 校刊美術史料: 寺院篇, Fujita Tsuneyo 藤田経世 (éd.), Tōkyō, Chuō Kōron Bijutsu Shuppan, 1972, vol. 3 (jomaki 上巻), p. 60

46. L'inscription est gravée sur la face incurvée du sakkan 檫菅 (ou satsu 檫), un élément en bronze de la flèche.

47. Les bâtiments de Yakushi-ji s'étendent sur 12 bō 坊 et ceux de Moto Yakushi-ji sur 6 bō. Voir Ōhashi, 1986 cité n. 33, p. 74-79. Sur Heijō Yakushi, voir Ōta Hirotarō 太田博太郎, Nanto shichidaiji no rekishi to nenpyō 南都 七大寺の歴史と年表, Tōkyō, Iwanami Shoten, 1979 p. 116-117; Yakushi-ji hakkenchōsa 薬師寺発掘調査, p. 8 ; et Tateno Kazumi 舘野和己, Kodaino Miyako 2. Heijō-kyo no jidai 古代の都2平城京の時代, Tōkyō, Yoshikawa kōbunkan, 2010, p. 91. McCallum, 2008, cité n. 6, indique par erreur 15 bō pour Yakushi-ji. Yakushi-ji disposait de 4 chō pour les bâtiments primitifs. Le Daikandai-ji de Fujiwarakyo disposait de 6 bō; le Daian-ji de Nara en a reçu 15.

48. Tombes royales de Songsan-ri (tombe 7). Les pièces étaient déposées sur l'une de deux plaques signalant 
I'acquisition du site par le roi contre paiement à la divinité de la Terre. Pour voir la tombe en ligne : http:// ryetour.net/songsan-ri-tombs-and-royal-tomb-of-kingmuryeong-송산리고분군과무령왕릉/.

49. Sūtra sur les vœux et les mérites originaux du Maître de Médecine Tathagata Lumière de Lapis Lazuli (ch. Yaoshi liulíguang rulai benyuan gongde jing 薬師瑠璃光 如来本願功徳経; Jp : Yakushi rurikō nyorai hongan kudoku kyō), généralement abrégé sous la forme Yakushikyō 薬師経. La traduction de Dharmagupta (615) n'est pas attestée dans le Japon ancien. Voir Raoul Birnbaum, The Medicine Master Buddha, Boulder, CO, Shambhala, 2003, et Suzuki, 2012, cité n. 6. Des spécialistes ont récemment formulé l'hypothèse que ruri 瑠璃 correspondait au beryl. Voir https://networks.h-net.org/node/6060/ discussions $/ 6173430 / \%$ E7\%90\%89\%E7\%92\%83-lapislazuli-beryl-or-something-else.

50. Birnbaum, 2003, cité n. 49, p. 62.

51. Birnbaum, 2003, cité n. 49, p. 68.

52. Pour voir les grottes en ligne : https://xts.uchicago.edu/. Pour voir la grotte Nord en particulier : https://asia.si.edu/exhibition/north-cave-at-northernxiangtangshan/\#jp-carousel-451788.

53. Pour des illustrations des deux, voir Igata Susumu 井形進, " Dazaifu-shiki onigawara kō: I-shiki A o chūshin ni » 大宰府式鬼瓦考: I 式Aを中心に一, dans Dazaifu shiseki hakkutsu 50 shūnen kinen ronbunshū kankōkai 大宰府史跡発掘50周年記念論文集刊行会, ed. Dazaifu no kenkyū 大宰府の研究, Tōkyō, Koshi Shoin, 2018, p. 561-574 (traduit en anglais, avec des adaptations, par Cynthea J. Bogel et Lindsey E. DeWitt : « Demon Roof Tiles: A Study of the Dazaifu Type Onigawara Style I-A », dans Journal of Asian Humanities at Kyūshū University [JAH-Q], vol. 4, mars 2019, p. 109-125).

54. Sur le motif de la racine de lotus, de l'empire Gupta à l'art bouddhique japonais, voir Andō Yoshika 安藤 佳香, Bukkyō shōgon no kenkyū: Guputa-shiki karakusa no tōden 佛教荘厳の研究: グプ夕式唐草の東伝, Tōkyō, Chūō Kōron Bijutsu Shuppan, 2003. Pour voir la naissance des yaksha dans les volutes des racines de lotus sur les parois de la grotte 17 d'Ajanta (entrée), voir p. 64. Selon Andō, ces volutes sont inspirées de celles qui apparaissent sous les piédestaux à motifs de lotus dans les sculptures bouddhiques de la période Gupta, ainsi que dans les représentations de vase floral (purnagha-ta) à l'eau jaillissante.

55. Ornementation du plafond et du pilier. Lotus avec Yakșa, hall, véranda, grotte 17 d'Ajanta. Voir Andō, 2003, cité n. 54, p. 64 et 65 (chap. V), illustrations en couleur.

56. Disponible en ligne à l'adresse suivante, URL : http://www.bl.uk/onlinegallery/onlineex/apac/other/ largeimage67844.html.

57. Des fragments, conservés au Museum für Asiatische Kunst de Berlin, Paysage de montagnes avec représentations de légendes bouddhiques, dans la grotte du Moulin à prières ( $\left.\mathrm{n}^{\circ} 114\right), 14 \mathrm{C}$, date : 391-427, peinture murale, $238 \times 236 \mathrm{~cm}$, voir : https://depts.washington.edu/silkroad/ museums/mia/im90a.jpg. Pour une vue d'ensemble de la collection : https://depts.washington.edu/silkroad/ museums/mia/kizil.html. Le site mentionne la publication de Su Bai, Ding Mingyi, Ma Shichang, Xiong Xi,
Xu Wanyin, Kezi'er shiku (The Cave Sanctuary of Kyzil), Tōkyō, Heibonsha, 1983-1985 et Pékin, Wenwu, 19891998, 3 vol., pl. 197.

58. Gurkeerat Singh, Textbook of Orthodontics, New Delhi, Jaypee Brothers Medical Publishers (P) Ltd., 2007, p. 320.

59. Par le maître chinois Yijing 義淨 (635-713), Nanhai jigui Neifazhuan. En anglais, voir Yijing, A Record of the Buddhist Religion as Practised in India and the Malay Archipelago (a.d. 671-695) by I-Tsing, translated by J. Takakusu, with a Letter from the Right Hon. Professor F. Max Müller, Taipei, Ch'eng Wen Pub. Co, 1970.

60. Voir par exemple en ligne, URL : https://en.dpm.org. cn/collections/collections/2015-05-08/3976.html. Pour une discussion plus détaillée et pour d'autres illustrations, voir aussi : Marc S. Abramson, Ethnic Identity in Tang China, Singapour, Institute of Southeast Asian Studies, 2014 ; Mark Edward Lewis, China's Cosmopolitan Empire: The Tang Dynasty, Cambridge, Mass., Belknap Press of Harvard University Press, 2012, en particulier p. 145178 ; et Shao-yun Yang, The Way of the Barbarians: Redrawing Ethnic Boundaries in Tang and Song China, Seattle, University of Washington Press, 2019.

61. Nancy Tingley, Andreas Reinecke, Arts of Ancient Viet Nam: from River Plain to Open Sea, Houston, Asia Society and the Museum of Fine Arts, 2009, p. 179 ("Champa: Riverine Polities, Ports of Call »). Tingley remarque que les habitants de Champa parlaient une langue austronésienne de la branche malayopolynésienne occidentale, celle qui s'est imposée dans les archipels d'Asie du Sud. Comme les Malais, les Chams étaient ethniquement austronésiens et leur principale langue écrite dans la période entre 200 et 700 de notre ère, où le cham était plus courant, était le sanscrit.

62. Martin Stuart-Fox, A Short History of China and Southeast Asia: Tribute, Trade and Influence, Crows Nest, N.S.W., Allen and Unwin, 2003, p. 43.

63. Voir Liu Xu 劉呴 et al., Jiu Tangshu 舊唐書 [Histoire des anciens Tang], Shanghai, Shangwu yinshuguan, 1936, chap. 197.5270 ; Feng Chengjun 冯承钧, Zhongguo Nanyang jiao tong shi 中国南洋交通史, Pékin, Shang wu yin shu guan, 1998, p. 51 ; Don J. Wyatt, The Blacks of Premodern China, Philadelphie, University of Pennsylvania Press, 2010, p. 145 et 167, n. 12.

64. Asanuma, 1993, cité n. 12, ill. 5 et 14, p. 53-71.

65. Trois des tuiles de la partie basse des deux pagodes (beaucoup ont été retrouvées à l'état de fragments) sont visibles à l'adresse suivante, URL : http://m.srbsm. co.kr/view.php?idx=28814. Rappelons que le temple de Sacheonwangsa a été construit pour dissuader les ambitions des Tang sur le royaume de Silla.

66. Il en est fait état dans le Nihon shoki. En anglais, voir Charlotte von Verschuer, "Japan's Foreign Relations 1200 to 1392 A.D.: A Translation from 'Zenrin Kokuhōki' », dans Monumenta Nipponica, vol. 54, $\mathrm{n}^{\circ}$ 1, Printemps 1999, p. 1-39, p. 19-20, et Bruce Batten, Gateway to Japan: Hakata in War and Peace, 500-1300, Honolulu, University of Hawaï Press, 2005, p. 25. et p. 145, n. 56, citant Naoki Kōjiō, Kodai Nihon to Chōsen, Chūgoku, Tōkyō, Kōdansha, 1988, p. 175-209.

67. Donohashi Akio 百橋明穂, Kodai hekiga no sekai: Takamatsuzuka, Kitora, Hōryū-ji Kondō 古代壁画の 
世界: 高松塚・キトラ・法隆寺金堂, Tōkyō, Yoshikawa Kōbunkan, 2010.

68. L'« emuseum » national de Tōkyō en donne quelques exemples aux adresses suivantes, URL : http://www. emuseum.jp/detail/100427? $x=\& y=\& s=\& d \_l a n g=f r \& s$ lang $=\mathrm{ja} \&$ word $=\% \mathrm{E} 7 \% 94 \% \mathrm{~B} 7 \% \mathrm{E} 5 \% \mathrm{~A} 5 \% \mathrm{~B} 3 \&$ class $=\& \mathrm{t}$ itle $=\& c \_e=\&$ region $=\&$ era $=\&$ cptype $=\&$ owner $=\& p o s=$ $1 \&$ num $=4 \&$ mode $=$ simple $\&$ century $=$ (en français) ; et https://asukanavi.jp/point.php?pid=49 (en japonais).

69. Nagaoka, 2005, cité n. 9, p. 26-27, citant Asanuma. Pour une synthèse sur l'influence des Emishi sur les monuments, voir Nara Kokuritsu Bunkazai Kenkyūjo [The Nara National Research Institute for Cultural Properties] et Asahi Shinbunsha jigyō honbu Ōsaka kikaku jigyōbu 朝日新聞社事業本部大阪企画事業部 (ed.), Asuka Fujiwarakyō-ten: Nara bunkazai kenkyūjo sōritsu 50 shūnenkinen 飛鳥・藤原京展: 奈良文化財 研究所創立50周年記念, Ōsaka, Asahi Shinbunsha, 2002, p. 64-67. La présence de sculptures est évoquée dans le passage du Nihon shoki concernant le règne de Saimei en 657, en 659, et en 660, cité dans Sotomura Ataru 外村中, “Asuka no Shumisenseki » 飛鳥の 須彌山石, dans Nippon Teien Gakkaishi, vol. 21, 2009, p. 1-14 (en ligne, DOI : https://doi.org/10.5982/jgarden. 2009.21_1).

70. Taihō 1, printemps, $1^{\text {er }}$ mois. 《大宝元年. 701正月 乙亥朔》 (Shoku Nihongi, vol. 2, Monmu Tenno 巻第二 文武天皇). Pour la signification de 《朝》, voir 《朝 貢 ». Parmi les meilleures sources sur le symbolisme des quatre animaux dans les tombes japonaises, sur les miroirs, et ailleurs, voir Donohashi, 2010, cité n. 68, en particulier p. 6-43. Voir aussi Tōkyō Kokuritsu Hakubutsukan [Musée national de Tōkyō] 東京国立 博物館 (ed.), Kitora kofun hekiga: tokubetsuten キトラ 古墳壁画: 特別展, Tōkyō, Tōkyō Asahi Shinbunsha, 2014, et Asuka Fujiwarakyō-ten..., 2002, cité n. 70, p. 166-167. En anglais, voir Ellen Van Goethem, «The Four Directional Animals in East Asia: A Comparative Analysis ", dans Asien- und Afrika-Studien der Humboldt Universitat zu Berlin, vol. 38, 2011, p. 201-216.

71. Les dignitaires envoyés à la cour des Tang avaient pour mission de prêter allégeance et de remettre un tribut tous les vingt ans. On pense que c'est à l'occasion de l'ambassade de 702 que le nom « Nihon» a été utilisé pour la première fois. Voir Nara Kokuritsu Hakubutsukan [Musée national de Nara] 奈良国立 博物館 (ed.), Dai Kentōshi-ten: Heijō Sento 1300-Nen Kinen 大遣唐使展 : 平城遷都1300年記念, Nara, Nara Kokuritsu Hakubutsukan, 2010, p. 381 (en japonais, p. 135).

72. On trouve des entrelacs de vigne sur les toits de tuile de temples coréens et dans des icônes bouddhiques de la Chine médiévale ainsi que dans leurs mandorles, par exemple dans la grotte 12 de Yungang 雲岡石窟 第 12 窟 (北魏 dynastie Wei du Nord), sur le mur sud de la salle principale 主室南壁; dans la grotte de Guyang 雲陽伯鄭長猷為亡父等造彌勒像 (datée de 501), sur le site des grottes de Longmen 龍門石窟 (pour voir les arches au-dessus des Bouddha Maitreya de la grotte de Guyang, URL : https://kknews.cc/culture/4o4gg8g. $\mathrm{html}$ ); et dans plusieurs des grottes de Mogao (209 et 322) qui remontent aux débuts de la dynastie Tang, à Dunhuang (conversations personnelles avec Lanying
Tseng, 13 novembre 2016). Les entrelacs de vigne apparaissent rarement dans l'art bouddhique japonais, et toujours au VII ${ }^{\mathrm{e}}$ siècle (on en trouve ainsi sur quelques tuiles et, à Hōryū-ji, sur un reliquaire 舎利容器, sur un ensemble de statues d'argile à la base de la pagode, et sur l'autel de Tachibana).

73. Hayashi Ryōichi, qui s'est intéressé à ces miroirs et à ces motifs de vigne, fait remonter ceux du piédestal de Yakushi-ji vers le milieu de la dynastie Tang. Voir Hayashi Ryōichi 林良一, "Yakushi-ji honzon daiza no budō karakusa mon » 薬師寺本尊台座の葡萄唐草文, dans Kokka 國華, vol. 810, 1959, p. 342-351 ; et Silk Road シルクロード, Tōkyō, Jijitsūshinsha, 1988, p. 177-198.

74. Par exemple dans les grottes du mont Maiji. Voir Fu Xinian, Traditional Chinese Architecture: Twelve Essays, Nancy S. Steinhardt (ed.), Alexandra Harrer (trad. angl.), Princeton, Princeton University Press, 2017. 